University of Nebraska - Lincoln

DigitalCommons@University of Nebraska - Lincoln

Faculty Publications from the Department of Engineering Mechanics

6-2009

\title{
Force Chains and Resonant Behavior in Bending of a Granular Layer on an Elastic Support
}

\author{
Kitti Rattanadit \\ University of Nebraska - Lincoln \\ Florin Bobaru \\ University of Nebraska - Lincoln, fbobaru2@unl.edu \\ Konlayut Promratana \\ University of Nebraska - Lincoln \\ Joseph A. Turner \\ University of Nebraska - Lincoln, jaturner@unl.edu
}

Follow this and additional works at: https://digitalcommons.unl.edu/engineeringmechanicsfacpub

Part of the Mechanical Engineering Commons

\footnotetext{
Rattanadit, Kitti; Bobaru, Florin; Promratana, Konlayut; and Turner, Joseph A., "Force Chains and Resonant Behavior in Bending of a Granular Layer on an Elastic Support" (2009). Faculty Publications from the Department of Engineering Mechanics. 56.

https://digitalcommons.unl.edu/engineeringmechanicsfacpub/56
}

This Article is brought to you for free and open access by the Mechanical \& Materials Engineering, Department of at DigitalCommons@University of Nebraska - Lincoln. It has been accepted for inclusion in Faculty Publications from the Department of Engineering Mechanics by an authorized administrator of DigitalCommons@University of Nebraska - Lincoln. 
Published in Mechanics of Materials 41:6 (June 2009), pp. 691-706; doi 10.1016/j.mechmat.2009.01.023

Copyright @ 2009 Elsevier Ltd. Used by permission. http://www.elsevier.com/locate/mechmat

Submitted January 18, 2009; published online January 30, 2009.

\title{
Force chains and resonant behavior in bending of a granular layer on an elastic support
}

\author{
Kitti Rattanadit, Florin Bobaru, Konlayut Promratana, and Joseph A. Turner \\ Department of Engineering Mechanics, University of Nebraska-Lincoln, W317.6 Nebraska Hall, Lincoln, NE 68588-0526, \\ Corresponding author - F. Bobaru, tel 402 472-8348, fax 402 472-8292, email fbobaru2@unl.edu
}

\begin{abstract}
In this paper we investigate the interaction between a granular layer and an elastic foundation using a coupled Discrete Element Method-Finite Element Method (DEM-FEM) computational model. We use this dynamics code to simulate quasi-static bending of the granular layer and we observe the changes taking place in the structure of the force chains for two cases: with and without rolling resistance. A reversal of the arches formed in the force chains leads to a bending resistance similar to that observed in dynamic experiments on resonant behavior under bending of a layer of sand in a container with an elastic bottom. The behavior of the force chains generated during bending may lead to enhanced mixing in vibrated granular media. In free vibration, the granular-layer/elastic-beam system behaves like a mass-loaded beam with no contribution to the stiffness from the granular layer, independent from the layer thickness and rolling resistance. This is observed to be due to the absence of the reversal of the force chain structures noticed in the quasi-static case when a push-up force bends the system upwards and the force chains rest against the middle of the beam and the side walls. Future studies are required for explaining the experimental observations related to the particle-size dependence of the bending stiffness in a granular layer as well as the resonant behavior of the system under forced bending vibrations.
\end{abstract}

\section{Literature review and problem description}

The discrete element method (DEM) has become a very popular tool for studying the micro-macro mechanical behavior of granular materials since first proposed by Cundall and Strack (1979). Applications of the DEM for modeling granular flow, and mixing and segregation, are now covering diverse fields such as powder technology, pharmaceutical industries, food industry and agriculture, geotechnical processes and civil engineering, mining, etc. (e.g., Antony, 2007; Campbell, 1997; Campbell, 2006; Cleary, 1998; Kudrolli, 2004; Lewis et al., 2005; Onate and Rojek, 2004; Sebastian and Luis, 2005; Tijskens et al., 2003; Yamane, 2004). The DEM is known as a "soft parti$\mathrm{cle}^{\prime \prime}$ method because the contacting particles are allowed to slightly deform (virtual overlap). The contacting point between particles is taken approximately at the center of the overlap region. In the DEM, the motion of an individual particle in the system is computed as follows: the particles' positions determine the overlap, which results in interaction forces (repulsive and/or attracting based on a particular mechanical model of interaction) that are integrated using the linear and angular momentum balance equations to compute the velocities of the particles. The new particles' positions are then updated using a particular integration scheme.

Early versions of DEM used simplified contact models, such as spring-dashpot (Cundall and Strack, 1979) but since then, other contact force models based on contact mechanics equations developed by, for example, Hertz (1882) for normal forces and Mindlin and Deresiewicz (1953) for tangential forces, have been used (see also Johnson, 1985). The choice for the contact force model depends very much on the specific geometry, material properties, and the gran- 
ular flow characteristics. Among the simplified models, the contact force represented by the linear spring-dashpot model is widely used (for some recent applications with this model see, e.g., Haff and Werner, 1986; Kuo et al., 2002; Cleary and Sawley, 2002; Schafer et al., 1996; Taguchi, 1992; Thompson and Grest, 1991; Zhang and Whiten, 1996). Advantages of this model are that it can be easily developed and applied for various particle shapes and geometries such as spheres, disks, ellipsoids, and the stiffness and damping parameters are analytically derived from the normal restitution coefficient and duration of time in contact (see, e.g., Schafer et al., 1996; Kruggel et al., 2007). Renzo and Maio (2004), however, point out that for this simple model to give reasonable results in simulations of granular flow, the parameters used need to be precisely evaluated. Among the nonlinear contact force models, Hertz theory is extensively used to compute normal forces while the Mindlin-Deresiewicz theory is used for tangential forces. The extension of Hertzian contact for cylindrical particles is given in Poritsky (1950) for cylinders and in Gerl and Zippelius (1999) for disks in 2D. Comparisons between using a contact-based model and a simplified model in modeling the mechanics of granular matter are reported in e.g., Renzo and Maio (2004) and Ji and Shen (2006). The nonlinear contact models have been used in, for example, modeling granular flow in a hopper (Langston et al., 1994), heap formation (Baxter et al., 1997), shot peening processes (Han et al., 2000a, 2000b) , contact of granular particle system to quantify inter-particle forces, velocity, and contact stresses (Thornton and Randall, 1988), impact of spherical particles with and without adhesion (Thornton and Yin, 1991).

The combined Discrete Element Method-Finite Element Method (DEM-FEM) was first proposed to study shot peening process by Petrinic (1996) in his doctorate work. Han et al. (2000a) gives results for 2D simulation of shot peening and explains in detail the treatment of possible contacts between a disk and the line segments of the finite elements. The shot is modeled by a discrete element while the impacted surface is modeled with finite elements. A review and equivalencies between various contact force models for small deformations is included. An extension of the model to 3D for shoot-peening applications is published in Han et al. (2000b). The combined DEM-FEM in 2D dynamic analysis of geomechanical problems is studied in Onate and Rojek (2004). This study involves fracture in cohesive granular material and plastic flow and wear in a cutting tool. Several examples are shown simulating rock cutting and tool wear, strip punch test and soil, and pipe interaction leading to pipe ovalization. The cutting tool is modeled by finite elements first, to simulate the plastic deformation, and then by discrete elements to model the wearing process. The soil or rock samples are modeled using discrete elements. Other versions of coupling between FEM and DEM are used in applications for reduced models of concrete structures in impact problems (see Frangin et al., 2006), introducing deformability in DEM particles for impact problems (Komodromos and Williams, 2004; Komodromos, 2005) and flow and compaction of irregular, randomly packed, particles to form a tabletted product (Gethin et al., 2006).

To the best of the authors' knowledge, there are no publications regarding the use of coupled DEM-FEM method to analyze the behavior of the force chains and the resonant frequencies of a granular-layer/elastic-beam system in bending deformation and vibration. In this paper we study the interaction (static and dynamic bending deformations) between a granular layer of disks loaded on top of a compliant elastic beam. The behavior of granular materials on vibrating plates has important applications in landmine detection (see e.g. Kang, 2006; Kang et al., 2007], and references therein). The problems studied in the present contribution are dimensionally-reduced versions of the system used in the experiments reported in Kang (2006) and Kang et al. (2007). In addition, recent simulation results (Promratana, 2008) have shown that shaking (in a container with elastic bottom) combined with forced vibration of the elastic bottom of the container can lead to dramatic enhancement of mixing and/or segregation in granular materials when compared to shaking in containers with inflexible bottoms.

The subsequent sections in this paper are arranged as follows: the equations of motion and the force model used in our simulations are described in Section 2; the 2D coupled DEM-FEM model for the granular-layer/elasticbeam system is given in Section 3. The 2D coupled DEMFEM implementation is validated with an ABAQUS FEM model in Section 4. In Section 5 we study the changes in the structures of the force chains and the resonance behavior of a granular-layer/elastic-beam (GLEB) system under slow-dynamic (quasi-static) bending deformation and under dynamic free-vibrations. Section 6 contains the conclusions and plans for future work.

\section{Equations of motion and the force model description in the 2D DEM model}

The governing equations of motion for a particle $i$, consisting of translational and rotational motions are described by the linear and angular momentum balance equations:

$$
\begin{aligned}
& m_{i} \frac{d \vec{v}_{i}}{d t}=\sum_{j}\left(\vec{F}_{i j}^{n}+\vec{F}_{i j}^{t}\right)+m_{i} \vec{g}=\vec{F}_{i}^{n}+\vec{F}_{i}^{t}+m_{i} \vec{g} \\
& I_{i} \frac{d \vec{\omega}_{i}}{d t}=\sum_{j}\left(\vec{R}_{i j}^{c} \times \vec{F}_{i j}^{t}-\vec{M}_{i j}^{r}\right)=\sum_{j}\left(\vec{R}_{i j}^{c} \times \vec{F}_{i j}^{t}\right)-\vec{M}_{i}^{r}
\end{aligned}
$$

where $\vec{v}_{i}$ and $\vec{\omega}_{i}$ are the translational and rotational velocity vectors of particle $i ; m_{i}$ and $I_{i}$ are mass and moment of inertia of particle $i ; \vec{F}_{i}^{n}$ and $\vec{F}_{i}^{t}$ are total normal and tangential forces acting on particle $i$ due to contact particle $j$; $m_{i} \vec{g}$ is the body force acting on particle $i \vec{R}_{i j}^{c}$ is the vector pointing from the contact point of the contacting pair $(i, j)$ to the center of particle $i$ with its magnitude equal to the particle radius $R_{i} ; \vec{M}_{i}^{r}$ is the total resisting moment acting on particle $i$ and caused by rolling friction. A schematic of forces acting on particle $i$ from particle-particle contact interaction is shown in Figure 1-left. 

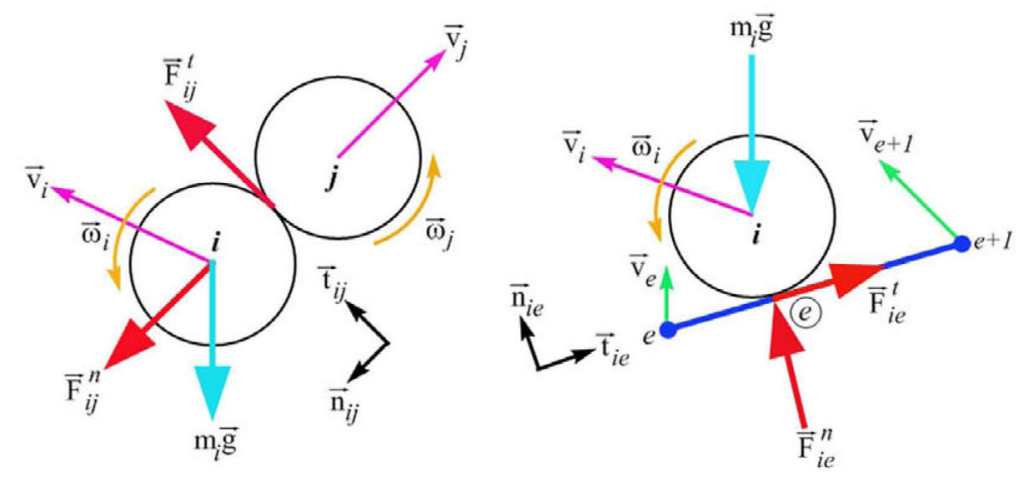

Figure 1. Schematic of the forces acting on particle $i$ : (left) due to contacting particle $j$ (particle-particle contact); (right) due to contacting beam element $e$ of nodes $e$ and $e+1$ (particle-beam in contact). Note that the resisting moment is not shown in the figure.

In the linear spring-dashpot model, the elastic constant $\left(K_{n}\right)$ is related to the contact time $\left(t_{c}\right)$ as $K_{n} \propto t_{c}{ }^{-2}$. Due to the fact that the evaluation of the contact time $\left(t_{c}\right)$ is difficult to conduct from the experiment, the related elastic constant value is somewhat questionable. For example, for the same physical phenomenon (fluidized granular bed), Tsuji et al. (1993) use in their simulations an elastic constant value of $800 \mathrm{Nm}^{-1}$, while $\mathrm{Xu}$ and $\mathrm{Yu}$ (1997) take a value of $50 \mathrm{KNm}^{-1}$. Renzo and Maio (2004) point out that using low values for $K_{n^{\prime}}$ results in large time steps that leads to excessive overlap. Since detailed contact measurements are not available, a more robust approach is to use a model that requires only material parameters of the particles, such as a contact-based model. For the contact interaction between the DEM particles (disks in 2D) as well as for the particle-beam interaction, we use the Hertz-type model for the elastic normal force as given in Gerl and Zippelius (1999) while for the elastic tangential force we use the simplified Mindlin and Deresiewicz model as shown in Johnson (1985). Such tangential force is also used in e.g., (Langston and Tuzun, 1994), (Langston et al., 1995) and (Baxter et al., 1997) for 2D analysis. Under "quasi-static" contact, the elastic normal and tangential forces are $F_{\text {elast }}^{n}=\pi E^{*} \xi / \ln \left(4 R^{*} / \xi\right)$ and $F_{\text {elast }}^{t}=$ $\left.\mu F_{\text {elast }}^{n}\left[1-\delta / \delta_{\max }\right)^{3 / 2}\right]$ where $\xi, \delta$ are the normal deformation and tangential displacement; $\mu$ is the sliding friction coefficient; $\delta_{\max }$ is the tangential displacement before sliding occurs and is related to the normal deformation by $\delta_{\max }=\mu E^{*} \xi / 4 G^{*}$ (see Langston et al., 1995); $E^{*}, G^{*}, R^{*}$ are the effective Young's modulus, effective shear modulus, and effective radius of the contact pair, respectively (see Equations (5), (7), and (8)). Since a cut-off for the tangential displacement is used, the elastic tangential force becomes $F_{\text {elast }}^{t}=\mu F_{\text {elast }}^{n}\left(1-\left[1-\min \left(\delta, \delta_{\max }\right) / \delta_{\text {max }}\right]^{3 / 2}\right)$. The elastic tangential force satisfies slipping $\left(\delta<\delta_{\max }\right)$ and sliding $\left(\delta \geq \delta_{\max }\right)$ conditions for the Mindlin and Deresiewicz model. The linear damping force component is computed from the product of the damping coefficient and the instantaneous relative velocity component where the damping coefficient is related to the critical damping $c_{c}=$ $2\left(m^{*} K\right)^{1 / 2}$ by use of damping ratio $\zeta$. Thus, $c=2 \zeta\left(m^{*} K\right)^{1 / 2}$, where $K=\partial F(S) / \partial S$ is the respective stiffness (Hookean spring); $S$ is the displacement corresponding to the con- tact force $F$ (for the normal force $S=\xi$ and the tangential force $S=\delta$ ); $\zeta$ is the damping ratio (assumed to be the same for both components) and is related to the normal restitution coefficient $\varepsilon_{n}$ by (see e.g., Asmar et al., 2002; Ji and Shen, 2006).

$$
\zeta=-\frac{\ln \left(\varepsilon_{n}\right)}{\sqrt{\pi^{2}+\ln ^{2}\left(\varepsilon_{n}\right)}}
$$

The total contact normal force including the elastic and damping components for small deformations $(\xi<R)$, which is implemented in our 2D coupled DEM-FEM model, becomes (see e.g., Kuninaka and Hayakawa, 2001),

$$
F_{i j}^{n}=\frac{\pi E^{*} h \xi}{\ln \left(4 R^{*} / \xi\right)}-2 \zeta \sqrt{\frac{\pi m^{*} h E^{*}}{\ln \left(4 R^{*} / \xi\right)}}\left(\vec{v}_{i j} \bullet \vec{n}_{i j}\right)
$$

where $F_{i j}^{n}$ is the magnitude of the contact normal force acting on particle $i$ due to contact particle $j ; \vec{v}_{i j}$ is the relative velocity at the contact point; $\vec{n}_{i j}$ is a unit vector normal to the tangent at the contact point of the pair $(i, j)$; $m^{*}$ is the effective mass of the contact pair; $h$ is the particle thickness respectively. The effective parameters for the contact pair $(i, j)$ can be calculated from the following relations,

$$
\begin{aligned}
\frac{1}{E^{*}} & =\frac{1-v_{i}^{2}}{E_{i}}+\frac{1-v_{j}^{2}}{E_{j}}, \\
\frac{1}{m^{*}} & =\frac{1}{m_{i}}+\frac{1}{m_{j}}, \\
\frac{1}{R^{*}} & =\frac{1}{R_{i}}+\frac{1}{R_{j}}, \\
\frac{1}{G^{*}} & =\frac{2-v_{i}}{G_{i}}+\frac{2-v_{j}}{G_{j}},
\end{aligned}
$$

$E, G, v$ are the Young's modulus, shear modulus (modulus of rigidity), and Poisson's ratio of a contacting component, respectively. The total contact tangential force accounting for the elastic and damping components for the simplified Mindlin-Deresiewicz model), which is implemented in our 2D coupled DEM-FEM model, becomes (see e.g., Langston and Tuzun, 1994; Langston et al., 1995; Baxter et al., 1997), 


$$
\begin{aligned}
F_{i j}^{t}= & -\operatorname{sgn}(\delta) \mu F_{i j}^{n}\left(1-\left(1-\frac{\min \left(\delta, \delta_{\max }\right)}{\delta_{\max }}\right)^{3 / 2}\right) \\
& -2 \zeta \sqrt{\frac{3}{2} \frac{m^{*} \mu F_{i j}^{n}}{\delta_{\max }}\left(1-\frac{\min \left(\delta, \delta_{\max }\right)}{\delta_{\max }}\right)^{1 / 2}}\left(\vec{v}_{i j} \bullet \vec{t}_{i j}\right)
\end{aligned}
$$

where $F_{i j}^{t}$ is the magnitude of the contact tangential force acting on particle $i$ due to contact particle $j$; $\vec{t}_{i j}$ is the unit tangential vector at the contact point of the pair $(i, j)$. Note that when sliding occurs $\left(\delta>\delta_{\max }\right)$ the tangential force is given by the Coulomb friction force with no damping component.

It has been reported in the DEM literature (e.g., Zhou et al., 1999; Zhu and Yu, 2006) that rolling resistance helps improve numerical stability and gives results that are more physical. We use a simple model for rolling resistance acting on particle $i$ due to rolling friction as in Johnson (1985):

$$
\vec{M}_{r}=\mu_{r} R_{i} F_{n} \hat{\omega}_{i}
$$

where $\hat{\omega}_{i}$ is the unit vector of angular velocity of particle $i ; \mu_{r}$ is the coefficient of rolling friction. Other options for modeling rolling resistance exist, like the one proposed in Brilliantov and Poschel (1998).

\section{Description of the 2D coupled DEM-FEM model for the granular-layer/elastic-beam (GLEB) system}

For a coupled 2D DEM-FEM simulation, two possible contact types called "full contact" and "partial contact" can occur during the contact between a discrete particle and a finite element. In full contact, as illustrated in Figure 1-right, the contact region between the particle and the beam element lies entirely within a single beam element, whereas the contact region touches more than one beam element in partial contact (not shown in Figure 1). The force model used for the discrete element/finite element interaction is the same as that used for the particle-particle interaction shown in the previous section. However, the "radius" of an element is taken as infinity and thus the effective radius of this contacting pair is the same as that of the discrete element. The relative velocity at the contact point between a particle and a finite element in the coupled DEM-FEM is given by (see e.g., Onate and Rojek, 2004),

$$
\vec{v}_{i e}=\left(\vec{v}_{i}+\vec{\omega}_{i} \times \vec{r}_{i}\right)-\left(\vec{v}_{e} N_{1}+\vec{v}_{e+1} N_{2}\right)
$$

where $\vec{v}_{i}, \vec{v}_{e}, \vec{v}_{e+1}$ are the absolute velocities of particle $i$ and beam element at node $e$ and $e+1$ of element $e ; N_{1}$ and $\mathrm{N}_{2}$ are the shape function at node $e$ and $e+1$ (local node 1 and 2), respectively. The contact forces are then calculated. Further details for computing the normal force for partial contact in 2D can be found in Han et al. (2000a).

Once the contact force acting on a particle is computed, the reaction force on the beam element is added up into the system force vector acting on the beam. The finite element formulation for the Euler Bernoulli beam leads to the system of second-order (hyperbolic) differential equations,

$$
[M]\{\ddot{U}\}+[K]\{U\}=\{F\}
$$

where $\{U\}$ is the beam nodal displacement vector, $[M]$ is the beam mass matrix, $[K]$ is the beam stiffness matrix, and $\{F\}$ is the force vector acting on the beam including the con- tact interactions with the discrete element and the applied force. We use the stable Galerkin time integration scheme (a particular type from the Newmark family methods) to approximate the new nodal beam displacement. This integration scheme reduces the differential equations to the system of algebraic equations (see e.g., Reddy, 2003).

$$
[\hat{K}]_{s+1}\{U\}_{s+1}=\{\hat{F}\}_{s+1}
$$

where

$$
\begin{aligned}
& {[\hat{K}]_{s+1}=[K]_{s+1}+a_{3}[M]_{s+1}} \\
& \{\hat{F}\}_{S+1}=\{F\}_{S+1}+[M]_{s+1}\left(a_{3}\{U\}_{S}+a_{4}\{\dot{U}\}_{s}+a_{5}\{\ddot{U}\}_{s}\right)
\end{aligned}
$$

Note that the nodal beam acceleration at the initial time step $(s=0)$ can be computed from the differential equation $\{\ddot{U}\}_{0}=[M]^{-1}\left(\{F\}_{0}-[K]\{U\}_{0}\right)$. The right-hand side vector in Equation (13) includes the initial and boundary conditions (see also Equation (13b)) and the external loads applied to the beam. The external loads are the contact interactions with the discrete elements and the applied load. At each time step, the nodal beam displacements are obtained by solving the system of linear equations, Equation (13). The accelerations and velocities for the beam are calculated using the following equations:

$$
\begin{aligned}
& \{\ddot{U}\}_{s+1}=a_{3}\left(\{U\}_{s+1}-\{U\}_{s}\right)-a_{4}\{\dot{U}\}_{s}-a_{5}\{\ddot{U}\}_{s} \\
& \{\dot{U}\}_{s+1}=\{\dot{U}\}_{s}+a_{2}\{\ddot{U}\}_{s}+a_{1}\{\ddot{U}\}_{s+1}
\end{aligned}
$$

where the coefficients used in Equations (13), (14), and (15) are $a_{1}=1.5 \Delta t, a_{2}=-0.5 \Delta t, a_{3}=1.25 /(\Delta t)^{2}, a_{4}=1.25 / \Delta t$ and $a_{5}=-0.375$.

Note also that the system mass and stiffness matrices are constant during simulation. The algorithm implemented in our 2D coupled DEM-FEM simulation has the following steps: input initial data (particle and box coordinates; particle, box, and beam dimensions; material parameters; initial kinematic quantities e.g. position, velocity, acceleration, etc); for a given time step we detect all contacts between particles, particle-beam element, particle-rigid wall to compute contact forces acting on the particle-discrete elements and beam-finite elements; we compute the total forces for all particles followed by all external forces acting on the beam including the contact interaction with the discrete elements and the applied load; we then perform time integration using the 5th order Gear algorithm (see e.g., Poschel and Schwager, 2005) for the motion of the discrete element particles and the Galerkin algorithm described above for the motion of the beam-finite elements; the positions and velocities are updated to continue to the next time step.

\section{Validation of the 2D coupled DEM-FEM with an ABAQUS FEM model}

For the simple case of a single particle positioned on the elastic beam we validate our coupled DEM-FEM code with a full finite element computation using ABAQUS. The system with the single particle sitting at the middle of a clamped-clamped slender Euler-Bernoulli beam is loaded by an oscillating force applied at the middle of 

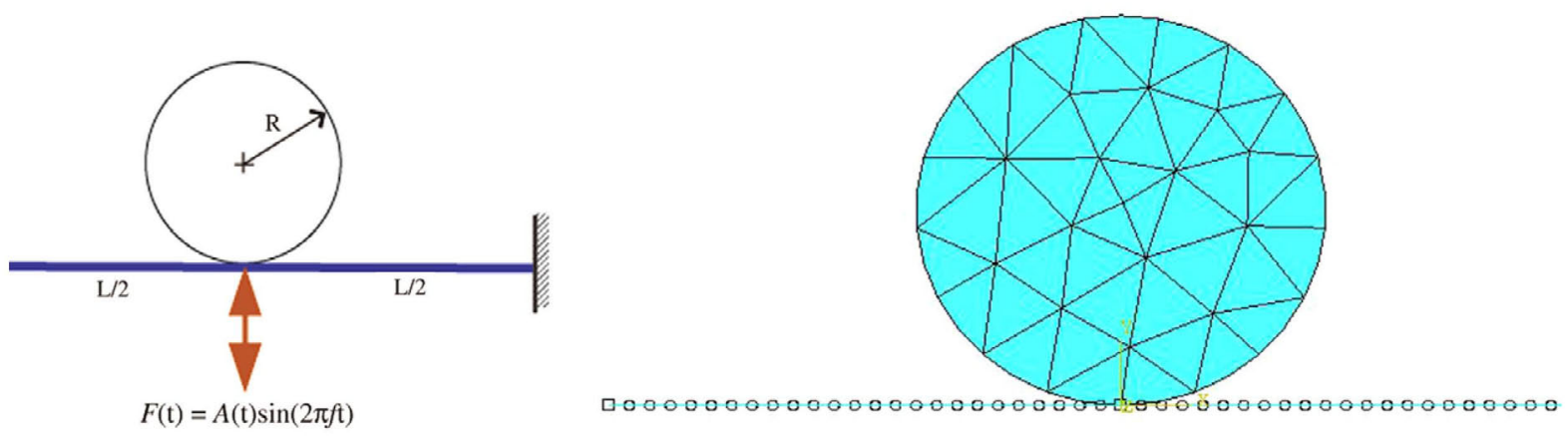

Figure 2. Particle/beam model (left) for validation of the DEM-FEM code using an FEM model in ABAQUS. A finite element discretization of the particle/elastic-beam system with ABAQUS (right).

the beam (see Figure 2). We monitor the position, in time, of the center of the particle. The oscillatory load is described by $F(t)=A(t) \sin (2 \pi f t)$, where $A(t)$ is a time-dependent amplitude which ramps linearly from $0 \mathrm{~N}$ at $t=0 \mathrm{~s}$ to $24.65 \mathrm{mN}$ at $t=0.5 \mathrm{~s}$ and stays constant afterwards until $t=1 \mathrm{~s}$, and $f$ is constant frequency equal to $5 \mathrm{~Hz}$. We intend this comparison for the cases when the particle stays in contact at all times with the beam because in our subsequent simulations we observe no separation. Moreover, if the particle starts separating from the beam, the impact and inertia of the large particle, will, eventually, result in large deformations of the beam and Euler-Bernoulli beam model is no longer a good approximation. Other combinations of amplitudes and frequencies can be selected for this validation but care has to be taken such that they do not lead to separation between the particle and the beam. The dimensions and material properties of the beam are as follows: length is $100 \mathrm{~mm}$, thickness is $0.25 \mathrm{~mm}$, depth is $0.25 \mathrm{~mm}$, Young's modulus equals $103 \mathrm{GPa}$, Poisson's ratio is 0.34 , and a density of $8575 \mathrm{~kg} / \mathrm{m}^{3}$ is used. For the particle, the parameters are: radius is $20 \mathrm{~mm}$, thickness is $0.25 \mathrm{~mm}$, Young's modulus is $70 \mathrm{GPa}$, Poisson's ratio 0.3, and density $1600 \mathrm{~kg} / \mathrm{m}^{3}$. The problem setup and the finite element discretization of the particle and elastic beam are shown in Figure 2.

To validate our coupled DEM-FEM code, a finite element model in ABAQUS is appropriate since, by discretizing both the particle and the beam using finite elements (2D plane-stress elements for the particle and beam elements for the beam, see Figure 2-right) and enforcing contact surfaces between them, the ABAQUS solution models the "true" contact, whereas our DEM-FEM code uses a Hertzian-type approximation for computing the contact forces. In ABAQUS we select "Hard contact" pressure-overclosure model for normal contact behavior and set the model for allowable separating after contact. The tangential contact behavior is defined by a penalty friction formulation with 0.3 of friction coefficient. In this formulation, elastic slip is allowable. In our coupled DEM-FEM model, we use cubic Euler-Bernoulli beamfinite elements with explicit time integration for the discretization of the elastic beam. In the ABAQUS model, the Euler-Bernoulli beam element B23 uses implicit integration. There is also the first-order shear deformation explicit element B21. Since, for the tests we run, the explicit solution is faster than the implicit one we choose to compare our DEM-FEM solutions with those from the ABAQUS explicit B21 element discretization. A convergence study for the modal analysis of elastic beam alone is performed and the ABAQUS and our FEM model show that using 50 elements produces the first resonant frequency with an error less than $0.1 \%$ compared to the analytical solution.

For the particle/elastic-beam system, the particle is meshed, in ABAQUS, using the 6-node triangular planestress 2-D elements (CPS6M). Under the forcing function $F(t)$ given above, a convergence study, in terms of the number of finite elements used to discretize the particle, is considered and the results are shown in Figure 3. The discretization with 45 elements is sufficient for a very good approximation of the converged result.

In Figure 4 we show the comparison between the solutions from our DEM-FEM code and ABAQUS solutions using 4-noded quad plane-stress elements (CPS4R) and 6noded triangular element (CPS6M). The match between our DEM-FEM solution and the ABAQUS solution using the quadratic triangular elements is excellent. The small difference noticed for the solution that uses the CPS4R can be attributed to the fact that these elements offer a lineartype approximation and the convergence to the exact solution will be lower than that of the CPS6M elements that use quadratic shape function. Because of their higher accuracy, we select the quadratic elements in the next test.

In the solutions above, the higher frequency response is due to the free vibration of the particle/elastic-beam system (which is about $18 \mathrm{~Hz}$ ). This effect disappears if the initial amount of external force applied is equivalent to the system's weight, to balance it (see Figure 5). The match between the ABAQUS solution and the DEMFEM solution is again perfect (for stiff particles). If a softer particle (for example if the particle Young's modulus $E<<100 \mathrm{MPa}$ ) is used under the same forcing frequency $(5 \mathrm{~Hz})$ and an amplitude that ramps linearly from the system's weight at $t=0 \mathrm{~s}$ to $0.025 \mathrm{~N}$ at $t=0.5 \mathrm{~s}$ and then stays constant, the particle starts rolling in the DEMFEM model (which results in the observed delay in Figure 5-right) while in ABAQUS the particle does not roll. This is likely due to the fact that the Hertzian contact 

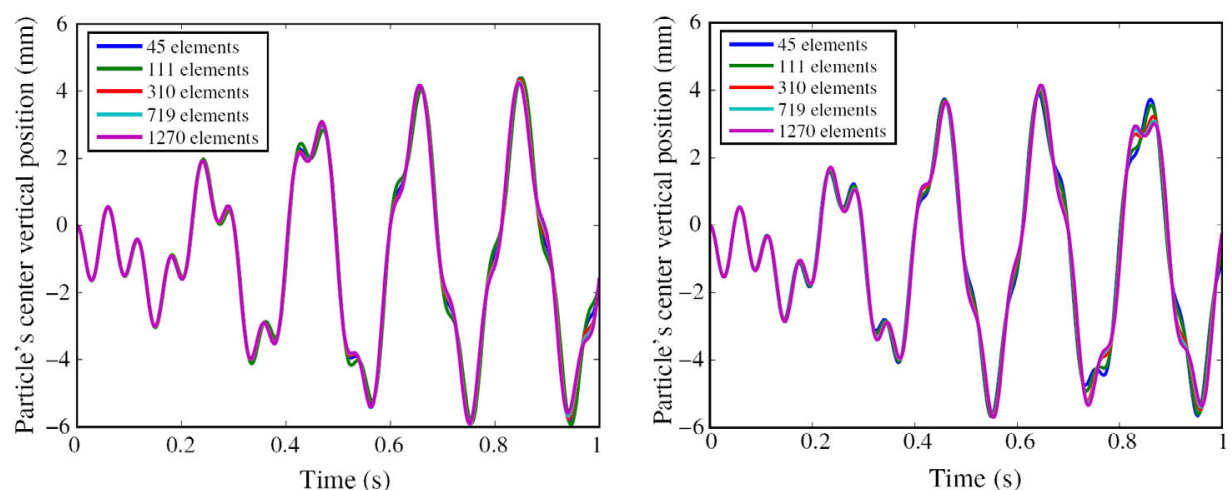

Figure 3. Convergence study for the ABAQUS model in terms of the number of elements used to discretize the particle. The forcing described in the text is applied to the particle/elastic-beam system and the position of the center of the particle is monitored. Using 6-noded triangular elements (CPS6M) and Young's modulus values of $E_{\mathrm{p}}=1 \mathrm{MPa}$ (left) and $E_{\mathrm{p}}=70 \mathrm{GPa}$ (right).

model, used in the DEM-FEM model, is no longer a good approximation of the elastic deformation of the very soft particle. Notice that here we did not use rolling resistance in the DEM-FEM simulations.

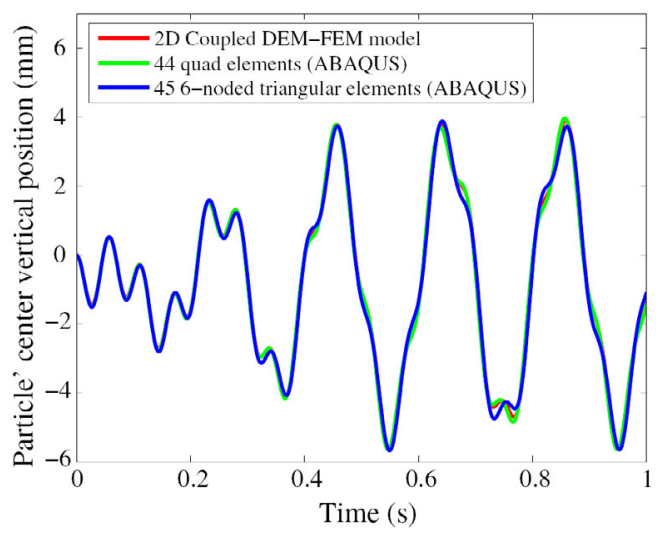

Figure 4. Comparison of the displacement of the particle's center using the DEM-FEM code and two types of elements (linear quads and quadratic triangular elements) in ABAQUS. The forcing is as described in the text and the particle's Young's modulus is $70 \mathrm{GPa}$.

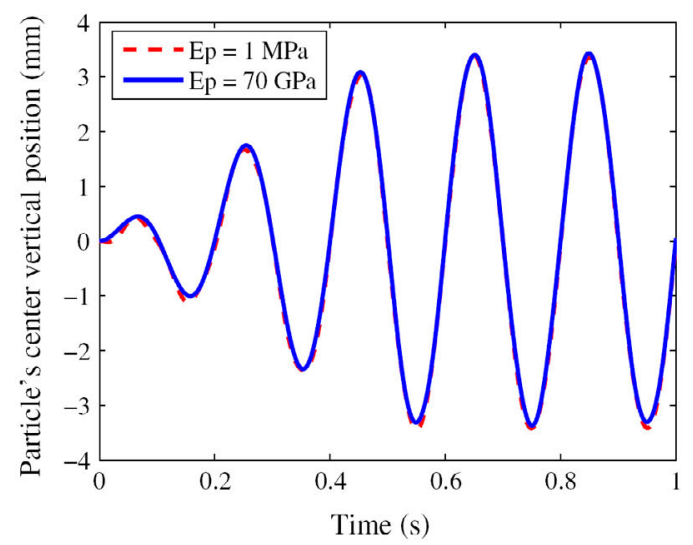

\section{Numerical results of the GLEB system subject to bending deformation and small vibration}

\subsection{Simulation setup and parameters}

In all subsequent simulations, a close packed layer of monodispersed particles is considered with the parameters and material properties summarized in Table 1 . The parameters for the elastic beam on top of which the granular particles rest are also contained in Table 1. The sidewalls are considered rigid, but when computing contact with the particles we use stiffness parameters for the sidewalls to be the same as those of the flexible bottom beam. In plotting the force chains we leave out forces that are 100 times smaller than the largest normal force during a specific simulation. In addition, for better size separation, we employ a logarithmic scale to compute the line thickness that represents the magnitude of the normal force in contact between particle--particle, particle-wall, or particle-finite element:

$$
L=\log \left[F_{i j}^{n}(e-1) / F_{\max }^{n}+1\right]
$$

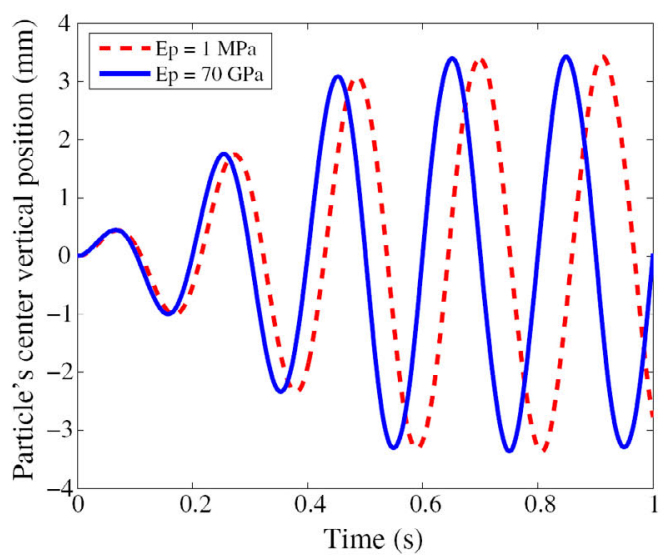

Figure 5. Vertical displacement of particle's center for the case when the applied forcing starts with an amplitude value equal to the system's weight. The ABAQUS solution for a stiff and a very soft particle (left) and the coupled DEM-FEM solution for the same particles (right). 
Table 1. Parameters used in the simulation.

\begin{tabular}{ll}
\hline Parameters and material properties & Value \\
\hline Beam & \\
Dimension (length, thickness, depth) & $100,0.25,0.25 \mathrm{~mm}$ \\
Number of discretized beam elements & 50 elements \\
Density & $8575 \mathrm{~kg} / \mathrm{m}^{3}$ \\
Young's modulus & $103 \mathrm{GPa}$ \\
Poisson's ratio & 0.34 \\
Particle & \\
Dimension (radius, thickness) & $0.625,0.25 \mathrm{~mm}$ \\
Number of particles (mass ratio $~ 2.37,4.73$, & $159,318,636,954$, \\
$\quad 9.47,14.20,35.50)$ & $2385 \mathrm{particles}$ \\
Density & $2600 \mathrm{~kg} / \mathrm{m}^{3}$ \\
Young's modulus & $1 \mathrm{GPa}$ \\
Poisson's ratio & 0.30 \\
Contacting interface of all contacts & \\
Normal restitution coefficient & 0.60 \\
Sliding friction coefficient & 0.30 \\
Rolling friction coefficient & 0.03 \\
\hline
\end{tabular}

where $L$ is the line-thickness; $F^{n}$ ij is the contact normal force between pair $(i, j) ; F_{\max }^{n}$ is the maximum normal force in the system during the entire simulation event; $e \approx 2.7183$. The $\log$ scale allows us to see forces that are several times smaller than the largest one plotted.

\subsection{Structure of force chains of the GLEB system subject to bending deformations}

The coupled 2D DEM-FEM model described in the preceding sections is used to study the structural changes in the force chains when the elastic beam, loaded by the granular layer, is deformed in bending by a push-up uniformly-distributed force, as shown in Figure 6-left. The magnitude of the applied force (per unit length) is shown in Figure 6-right as a function of time. We investigate the structural changes that take place in the force chains during quasi-static bending of the clamped-clamped elastic beam loaded by the granular layer.

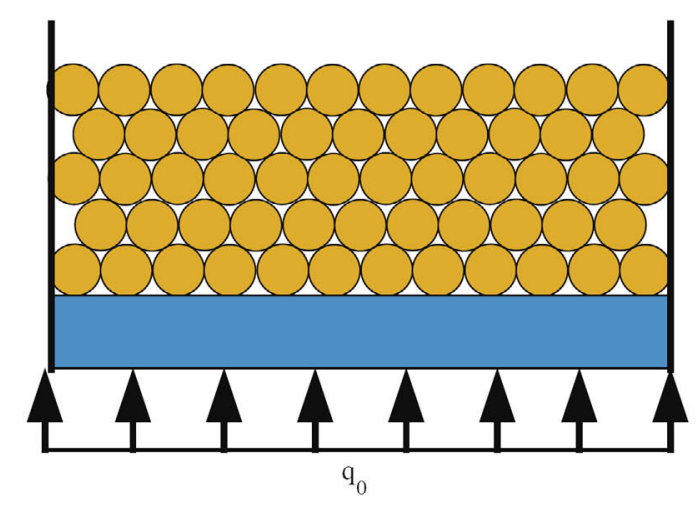

We let the system relax under its own weight for $1 \mathrm{~s}$ (see Figure 6). After reaching the equilibrium position, the applied force linearly increases for $2 \mathrm{~s}$ (to reduce transient effects that would be caused by an applied force that is discontinuous) and stays constant for another $2 \mathrm{~s}$. We test 2 cases: with and without rolling resistance (rolling resistance is applied to all contacts). In this section, we focus on the structure of the force chains, and we choose the thickest layer (mass ratio $\sim 35.50$ ) to represent these forces because for these thicker granular layers we can easier observe the richness of these structures. A discussion of the influence of the layer's thickness on the structure of the force chains is given in Rattanadit (2009) and Rattanadit et al. (2009).

In Figure 7, we show the structural changes of the force chains taking place in the granular material during the bending deformation caused by the uniformly-distributed force pushing upward on the beam, for the case when rolling resistance is considered. The first snapshot (at $0.025 \mathrm{~s}$ ) shows the formation of arches with the layer resting mostly on the left and right end parts of the beam while the middle is under very low pressure. This confirms the results in Baxter et al. (1997), which discusses the formation of such arches and transmittal of the force chains in granular piles on rigid supports as well as those in Zhou et al. (2003) where the pressure dip under wedge-sand piles on deflected bases is analyzed. Here, on the elastic foundation, these results are preserved. Notice that in our case we have side walls which are not present in Baxter et al. (1997) or in Zhou et al. (2003). The low pressure region in the middle and bottom of the granular layer is notable. The system reaches equilibrium before $1 \mathrm{~s}$, at which instant the force chains look similar to those at $0.025 \mathrm{~s}$ (see figure at $1.0 \mathrm{~s}$ ). As the push-up force is increasing in amplitude (after $1 \mathrm{~s}$ ), the force chain arches start to be destroyed (see figures at $1.3 \mathrm{~s}$ and $1.35 \mathrm{~s}$ ) and a $\mathrm{V}$-shaped area of low contact forces is formed in the center at around $1.375 \mathrm{~s}$. We also notice that the force chains and pressures concentrate around the two bottom corners of the granular layer. With further upward bending of the granular layer, the system is developing force chains that

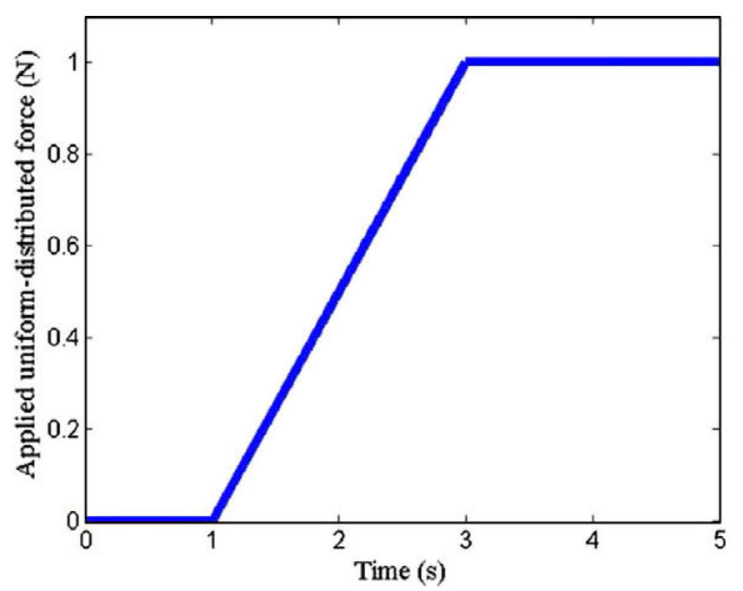

Figure 6. An elastic-beam/granular-layer system subjected to the applied uniformly-distributed force (left). The time-variation of the applied force per unit length $q_{0}$ (right). 
a

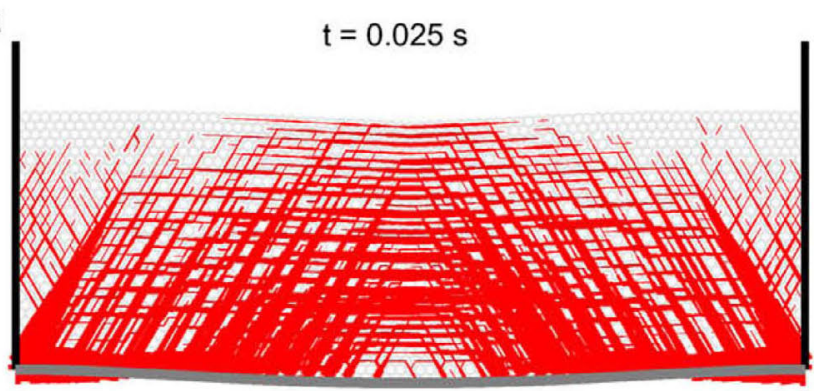

C

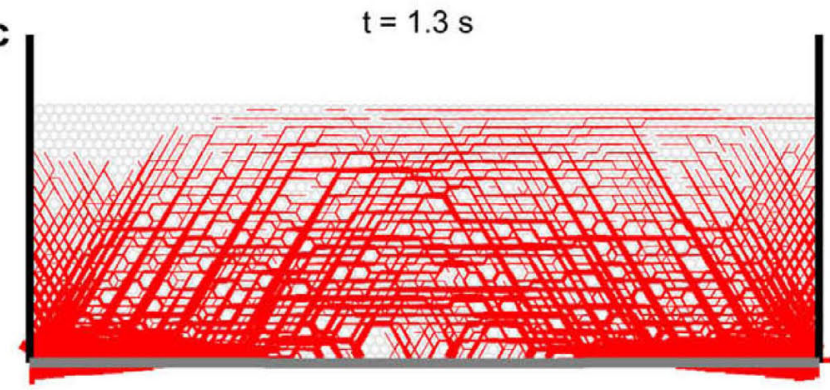

e

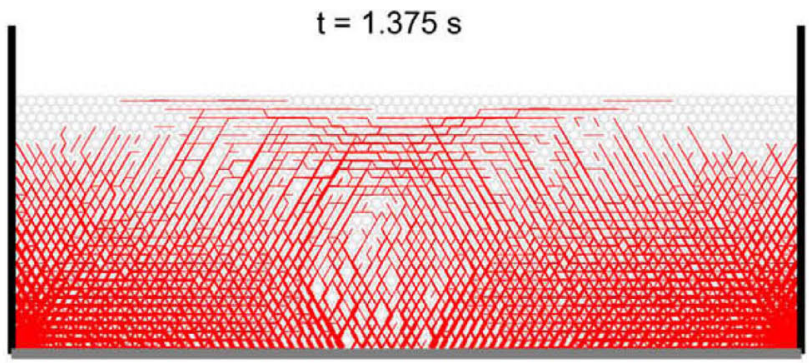

g

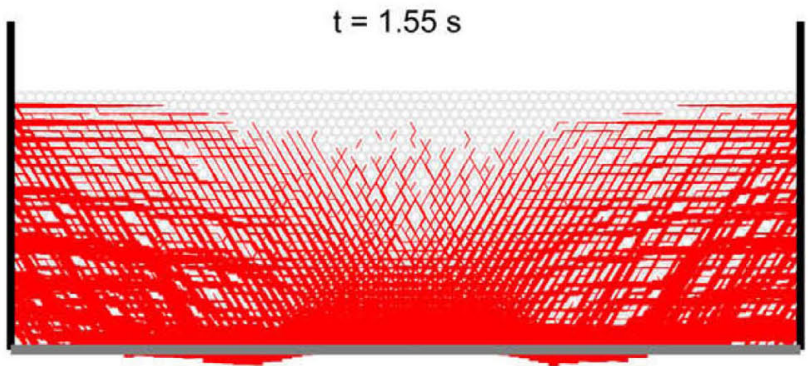

b

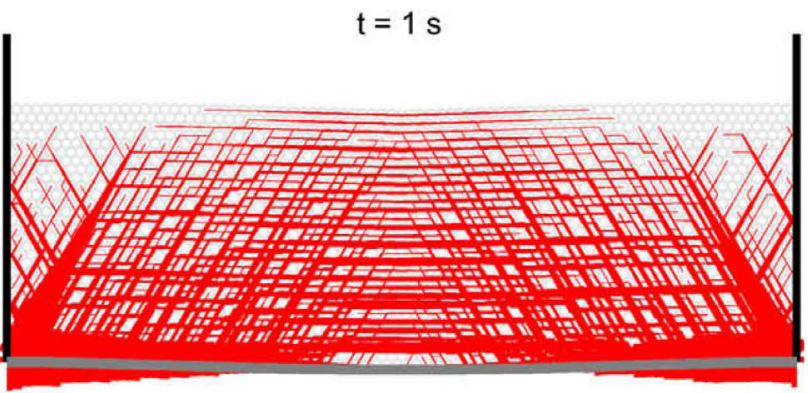

d

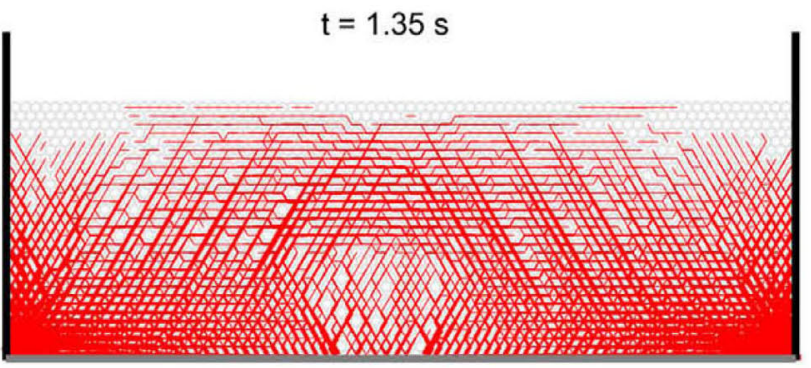

f

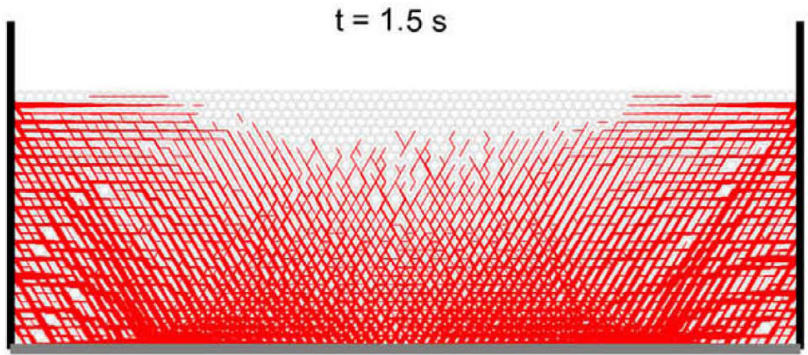

h

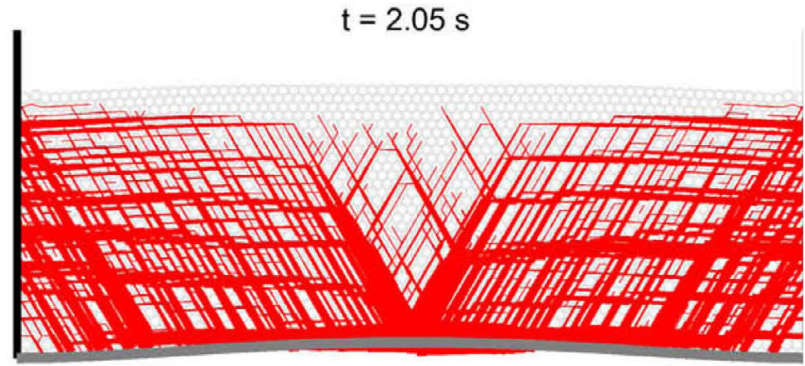

Figure 7. Snapshots of the contact force chains (shown in red) of the granular-layer/elastic-beam system under bending deformation with rolling resistance. Snapshots taken at $t=0.025,1.0,1.3,1.35,1.375,1.5,1.55$, and $2.05 \mathrm{~s}$. The particles, walls, and beam are drawn in light gray, black, and gray, respectively.

start pushing against the side-walls and the resting regions on the beam start moving towards the center (see figures at $1.5 \mathrm{~s}$ and $1.55 \mathrm{~s})$. As the push-up force increases, the beam bends upwards and the force chains in the granular layer form an "inverted arch" that pushes against the walls and rests against the middle of the beam (see figure at $2.05 \mathrm{~s}$ ). The pressure at the corners of the granular layer is also reduced. The force continues to increase until $t=3 \mathrm{~s}$ but the structure of the force chains no longer changes from that shown at $t=2.05 \mathrm{~s}$. A particularly interesting feature is the $\mathrm{V}$-shaped region of low pressure bounded by strong force chains seen in the last picture in Figure 7 . The behavior of the force chains in the bending process of the granular layer has important consequences in enhanced mixing and/or segregation as shown in Promratana (2008).

Without rolling resistance, the structural changes of the force chains evolved during bending deformation are shown in Figure 8. The overall formation of the force chain structures is similar to the case of rolling resistance. However, the rolling resistance induces higher symmetry and stability of the force chain structures, and enhances the magnitudes of the normal contact forces. We conclude that rolling resistance gives results that are more realistic. Note also the comments in Zhou et al. (1999) where rolling resistance is used in simulations of spheres heap formation. 

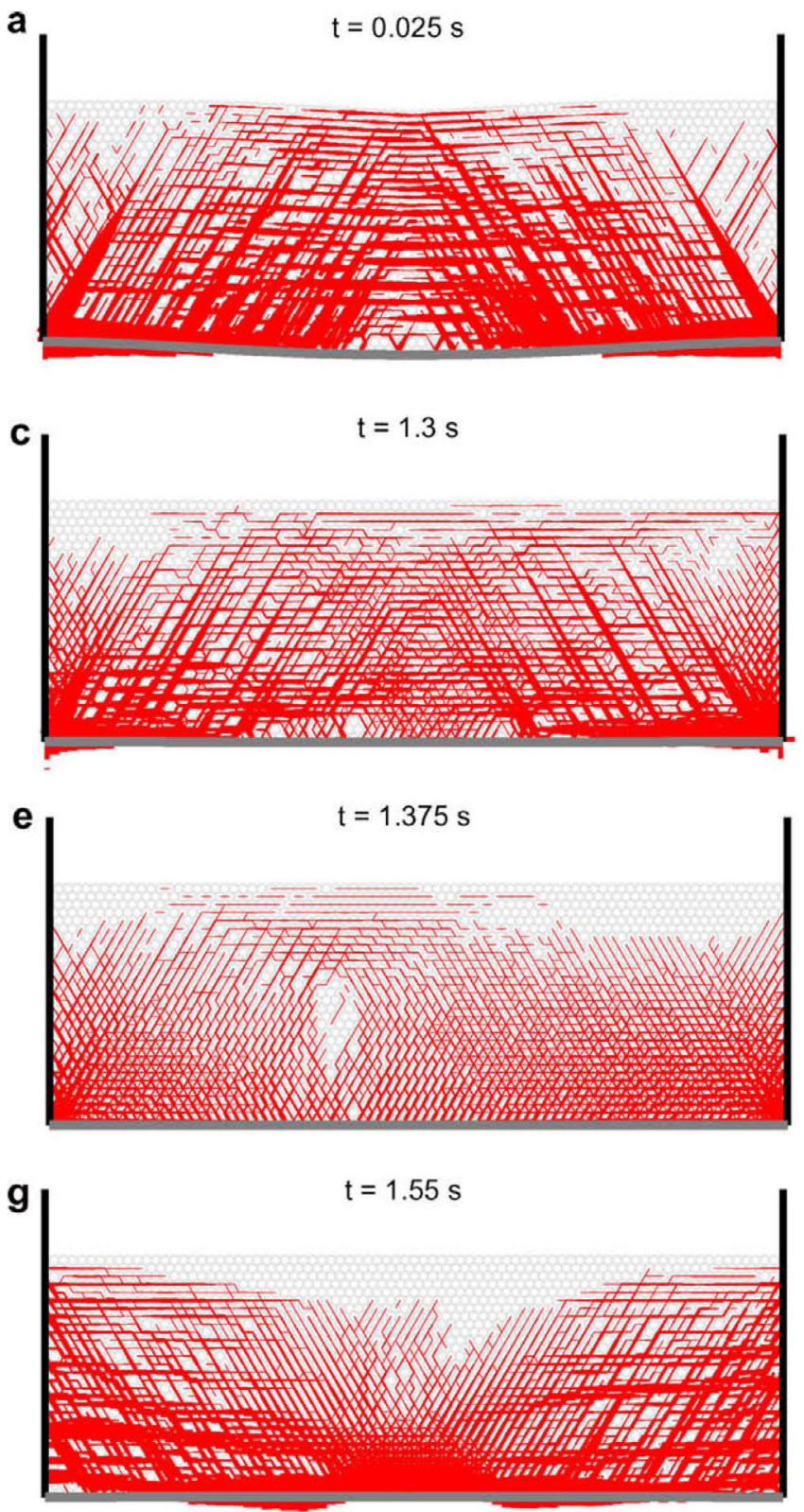
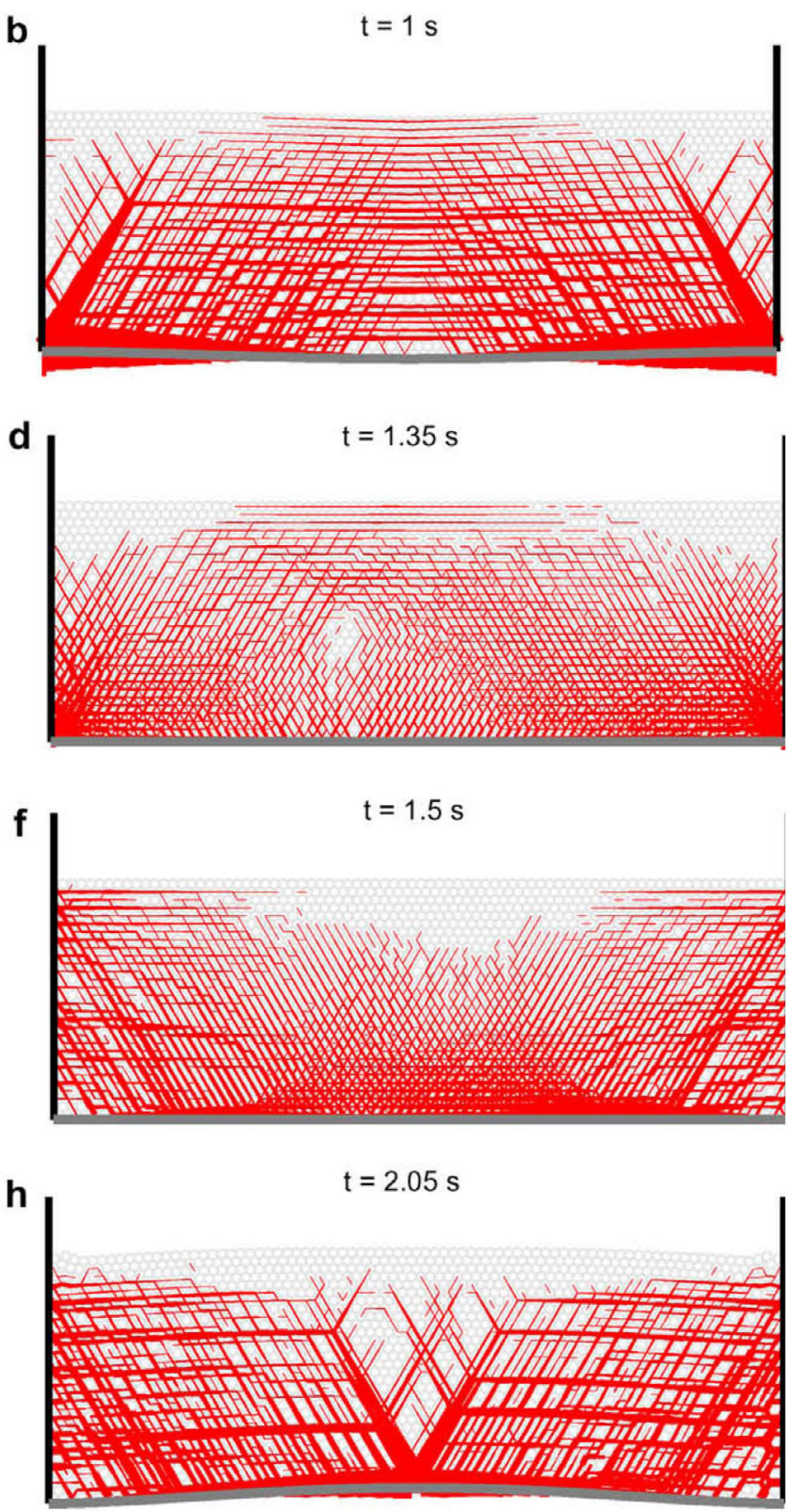

Figure 8. Snapshots of the contact force chains (shown in red) of the GLEB system under bending deformation without rolling resistance. Snapshots taken at $t=0.025,1.0,1.3,1.35,1.375,1.5,1.55$, and $2.05 \mathrm{~s}$. The particles, walls, and beam are drawn in light gray, black, and gray, respectively.

So far, we observed the variation of the force chain structure during quasi-static load (bending deformation). In the next section, we study the resonant behavior of the system and show a qualitative comparison with dynamic experimental results conducted in 3D where a circular plate is loaded on top by the granular layer (see Kang et al., 2007).

\subsection{Resonance behavior and effective bending stiffness of the granular layer}

In this section, we extract the natural bending frequencies of the GLEB system using an analogy with a two-layer composite beam system which we call the "EquivalentComposite-Beam" (ECB) (see Figure 9-left). The granular layer of the GLEB system (see Figure 6-left) is replaced by the top beam in Figure 9 whose Young's modulus $\left(E_{1}\right)$ is computed so that the ECB system, under the same loading and boundary conditions as the GLEB, gives the same mid-point deflection as that computed from the bending of the GLEB system reported in the previous section. The top beam in the ECB has the same thickness, density, depth as the granular layer. The bottom beam in the ECB system is identical as that used in the GLEB (clampedclamped ends).

We select five different thicknesses corresponding to five granular layer thicknesses (or mass ratios of 2.37, $4.73,9.47,14.20$, and 35.50) and compute the mid-point deflections from the DEM-FEM simulations for the GLEB system are shown in Figure 9 (right) for the cases with 

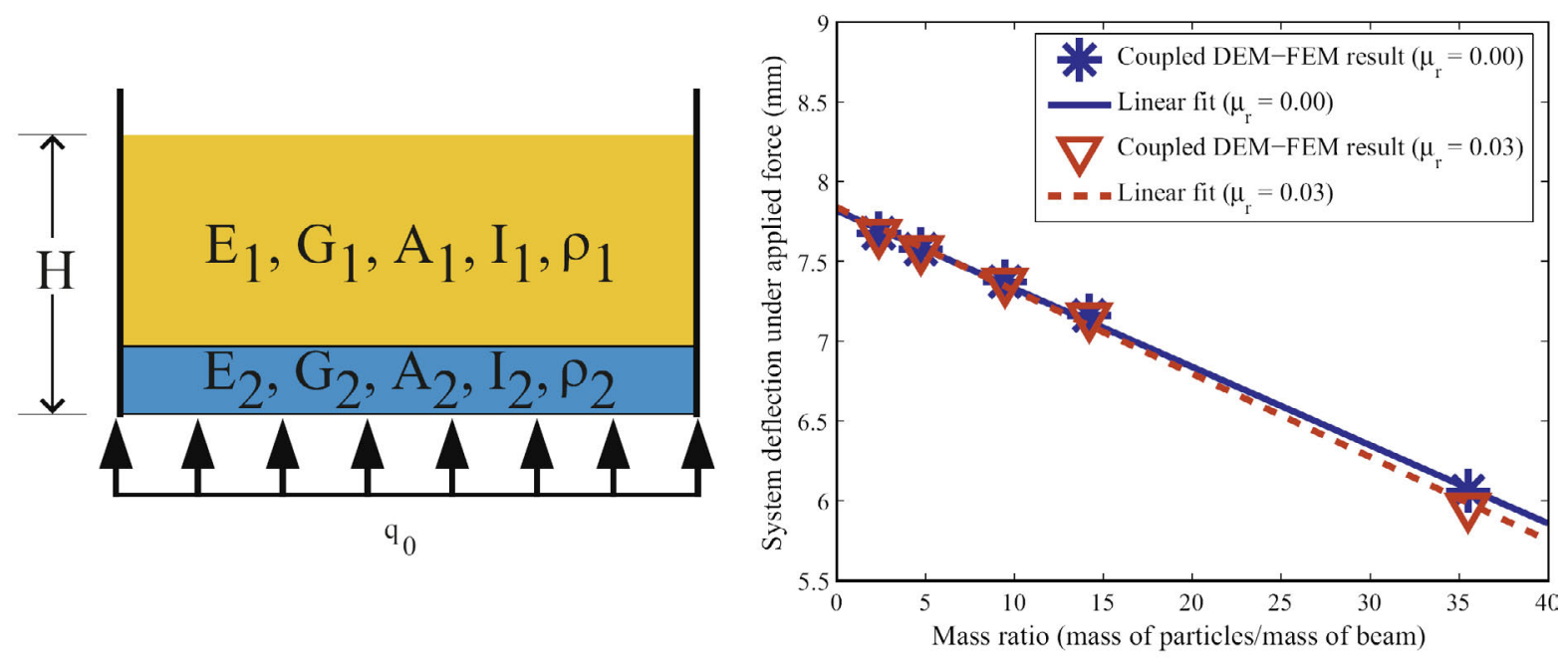

Figure 9. The Equivalent-Composite-Beam (ECB) system (left). Total deflections obtained from the coupled DEM-FEM simulation along with a linear curve fit for five different thicknesses (or mass ratios) of the granular layer. Results are shown (right) for two cases: with (red) and without (blue) rolling resistance.

and without rolling resistance. A linear curve fit is used in the calculations below to evaluate the mid-pint deflections at all other intermediate thicknesses. The parameters used for the top beam in the ECB system are given by (see Table 1): $A_{1}=H_{1} b_{1}, I_{1}=b_{1} H_{1}^{3} / 12, \rho_{1}=M_{1} / V_{1}$, $V_{1}=A_{1} L, v_{1}=0.30$ where $A_{1}$ is the cross-sectional area; $I_{1}$ is the cross-sectional moment of inertia; $\rho_{1}$ is the mass density; $M_{1}$ is the mass; $V_{1}$ is the volume; $H_{1}$ is the thickness; $b_{1}$ is the depth; $L$ is the length; $v_{1}$ is the Poisson's ratio of the top beam, respectively. It should be noted that the selection of the Poisson's ratio is reasonable because its influence on the results, for this kind of deformation (bending), is minimal (see computations and discussions in Kang et al., 2007).

To find the Young's modulus of the top beam in the ECB we solve a nonlinear equation (see Equation (17)) that connects the bending stiffness and the deflection under a uniformly-distributed force for a composite beam. This Young's modulus value is then used to compute the first bending resonant frequency for the ECB which will be considered as the first natural bending resonant frequency of the GLEB system. The details of this computation are described below.

For a given thickness, the Young's modulus $\left(E_{1}\right)$ is computed from the nonlinear Equation (17) (see $\mathrm{Xu}$ and $\mathrm{Wu}, 2007)$. For the clamped-clamped (CC) boundary conditions on both beams (Figure 9-left), the mid-point deflection of the ECB system under a uniformly-distributed load is given by,

$$
\Delta=\frac{q_{0} L^{4}}{384 \overline{E I}}\left[1+\left(\beta^{2}-1\right) f_{\mathrm{CC}}(\alpha L)+48\left(\frac{H}{L}\right)^{2} \frac{\overline{E I}}{H^{2} \overline{\kappa A G}}\right]
$$

Here $\Delta$ is the maximum (mid-point) deflection; $q_{0}$ is the uniformly-distributed load; $L, H=H_{1}+H_{2}$ is the length and total height of the two beams; $H_{i}(i=1,2)$ is the beams height; $\overline{E I}=\sum E I+E_{1} A_{1} E_{2} A_{2} h^{2} /\left(E_{1} A_{1}+E_{2} A_{2}\right)$ is the flexural stiffness of the composite beam where $\sum E I=E_{1} I_{1}+E_{2} I_{2}$ and $h$ is the distance between the beam centroids; $k A G=$ $\kappa_{1} G_{1} A_{1}+\kappa_{2} G_{2} A_{2}$ where $\kappa_{i}(i=1,2)$ is the shear correctors of the Timoshenko's beam theory depending on the shape of the cross-section of the two beams; $\alpha^{2}=k_{s}\left(1 / E_{1} A_{1}+1 /\right.$ $\left.E_{2} A_{2}+h^{2} / \sum E I\right)$ is the composite parameter related to the stiffness of the shear connector $k_{s^{\prime}} \beta^{2}=\overline{E I} / \sum E I ; E_{i^{\prime}} G_{i^{\prime}} A_{i^{\prime}}$ $I_{i}(i=1,2)$ denote Young's modulus, shear modulus, crosssectional area, and second moment of area of the two beams, respectively. Note that the correction of the partial interaction (interlayer slip between the contact surfaces) and shear deformation are given in the second and third terms in the brackets in Equation (17), where

$$
\begin{aligned}
f_{C C}(\alpha L)= & \frac{48}{\alpha^{2} L^{2}}-\frac{192 \sinh (\alpha L / 2)}{\alpha^{3} L^{3}} \\
& +\frac{192 \operatorname{coth}(\alpha L / 2)[\cosh (\alpha L / 2)-1]}{\alpha^{3} L^{3}}
\end{aligned}
$$

Also, the CC boundary conditions on the top beam is a reasonable assumption here because using rolling boundary conditions on the top beam has a minor effect within our deformation regime of interest as it was shown in Kang et al. (2007).

The results of Kang et al. (2007) for the match between a granular layer and a composite-plate elastic system, give us an initial guess for the Young's modulus value, for each granular layer thickness, used in the nonlinear solver (Matlab's "fzero" function) employed in the solution of Equation (17). We obtain Young's modulus values in the range of $8 \mathrm{MPa}$ (for thinner layers) decreasing almost monotonically with the thickness layer to $5 \mathrm{MPa}$ (for thicker layers), for the case when rolling resistance is considered, and $8 \mathrm{MPa}$ (for thinner layers) decreasing to $3 \mathrm{MPa}$ (for thicker layers) when rolling resistance is absent. These values are in similar range with those obtained from the experiments of Yanagida et al. (2003) and Kang et al. (2007) and they are significantly lower than those expected from wave speed measurements (Johnson, 1985; Oelze et al., 2002). Note, however, that the trends in terms of increased layer thickness are op- 
posite to those measured and shown in Figure 7 of Kang et al. (2007). The reason is likely due to the fact that the particle-size dependence of the (bending) stiffness measured from the resonant frequencies in the dynamic experiments of Kang et al. (2007) is much higher than that given by analytical effective-medium models. This discrepancy is yet to be fully explained. Our quasi-static simulations match the particle-size dependence or effective-media models in 2D (see Rattanadit, 2009; Rattanadit et al., 2009).

Next, we obtain the resonant frequencies of the ECB system by solving the characteristic equation of free vibration (see $\mathrm{Xu}$ and $\mathrm{Wu}, 2007$ ) given by:

$$
\left|\begin{array}{lll}
A_{C 1}(0) & A_{C 2}(0) & A_{C 3}(0) \\
A_{C 1}(L) & A_{C 2}(L) & A_{C 3}(L)
\end{array}\right|=0
$$

where

$$
\left[A_{C 1}(x)\right]=\left[\begin{array}{cc}
\sin \left(k_{1} x\right) & \cos \left(k_{1} x\right) \\
\left(-g_{\mathrm{C} 1} k_{1}^{3}+g_{\mathrm{C} 2} k_{1}\right) \cos \left(k_{1} x\right) & \left(g_{\mathrm{C} 1} k_{1}^{3}-g_{\mathrm{C} 2} k_{1}\right) \sin \left(k_{1} x\right) \\
\left(k_{1}^{5}-g_{\mathrm{C} 3} k_{1}^{3}\right) \cos \left(k_{1} x\right) & -\left(k_{1}^{5}-g_{\mathrm{C} 3} k_{1}^{3}\right) \sin \left(k_{1} x\right)
\end{array}\right],
$$

$$
\begin{aligned}
{\left[A_{C i}(x)\right] } & =\left[\begin{array}{cc}
\sinh \left(k_{i} x\right) & \cosh \left(k_{i} x\right) \\
\left(g_{C 1} k_{i}^{3}+g_{C 2} k_{i}\right) \cosh \left(k_{i} x\right) & \left(g_{C 1} k_{i}^{3}+g_{C 2} k_{i}\right) \sinh \left(k_{i} x\right) \\
\left(k_{i}^{5}+g_{C 3} k_{i}^{3}\right) \cosh \left(k_{i} x\right) & \left(k_{i}^{5}+g_{C 3} k_{i}^{3}\right) \sinh \left(k_{i} x\right)
\end{array}\right](i=2,3) \\
g_{C 1} & =\alpha^{2} \gamma_{3}\left(\gamma_{5}+1\right) \\
g_{C 2} & =\alpha^{2} \beta^{2}+\left(\alpha^{2} \gamma_{3}-2 \alpha^{2} \beta^{2} \gamma_{3}-2 \beta^{4}\right) \gamma_{1} \gamma_{3} \omega^{2}
\end{aligned}
$$

$$
\begin{aligned}
g_{C 3}= & -\alpha^{2}-\beta^{2} \gamma_{6} /\left(\gamma_{5}+1\right)+\gamma_{1} \gamma_{3} \omega^{2} /\left(\gamma_{5}+1\right) \\
& +\beta^{2} \gamma_{1} \gamma_{2} \omega^{2}+\alpha^{2} g_{C 4} / g_{C 2} \\
g_{C 4}= & \alpha^{2}-\beta^{2} \gamma_{1} \gamma_{3} \gamma_{4} \omega^{4}-\left(\alpha^{2} \gamma_{3}+\beta^{2}\right) \gamma_{1} \gamma_{3} \omega^{2}, \\
\gamma_{1}= & \overline{\rho A} / \overline{E I}, \quad \gamma_{2}=\overline{\rho I} / \overline{\rho A}, \quad \gamma_{3}=\overline{E I} / \overline{\kappa A G}, \\
\gamma_{4}= & \overline{\rho l} / \overline{\kappa A G}, \quad \gamma_{5}=F / \overline{\kappa A G}, \quad \gamma_{6}=F / \overline{E I}
\end{aligned}
$$

Here, $\omega$ is the resonant frequency of the composite beam; $F$ is the applied axial force (where $F=0$ in our case); $\overline{\rho A}=$ $\rho_{1} A_{1}+\rho_{2} A_{2} ; \overline{\rho I}=\rho_{1} I_{1}+\rho_{2} I_{2} ; \rho_{i}(i=1,2)$ is the mass density of the beams; $\pm k_{i}(i=1,2,3)$ are the six eigen-roots of the following equation:

$$
\begin{aligned}
& {\left[\gamma_{5}+1\right] k^{6}+\left[\beta^{2} \gamma_{1} \gamma_{2}\left(\gamma_{5}+1\right) \omega^{2}+\gamma_{1} \gamma_{3} \omega^{2}\right.} \\
& \left.\quad-\alpha^{2}\left(\gamma_{5}+1\right)-\beta^{2} \gamma_{6}\right] k^{4}+\left[\beta^{2} \gamma_{1} \gamma_{4} \omega^{4}-\beta^{2} \gamma_{1} \omega^{2}\right. \\
& \left.-\alpha^{2} \gamma_{1} \gamma_{3} \omega^{2}-\alpha^{2} \gamma_{1} \gamma_{2}\left(\gamma_{5}+1\right) \omega^{2}+\alpha^{2} \gamma_{6}\right] k^{2} \\
& \quad+\left[-\alpha^{2} \gamma_{1} \gamma_{4} \omega^{4}+\alpha^{2} \gamma_{1} \omega^{2}\right]=0
\end{aligned}
$$

The resonant frequency of the ECB system (or, equivalently, of the GLEB system), normalized by the frequency of the bottom beam alone, for various thicknesses of granular layer under the bending deformation is shown in Figure 10-left. For a qualitative comparison, in Figure 10, we also show the 3D experimental results on sand layers loaded on a circular plate (Kang et al., 2007). Note that the model for contact forces used in our simulations correspond to the 2D Hertzian contact, and the scaling with the particle radius in 2D (disks) differs from that in 3D (spheres). The experimental data in Kang et al. (2007) was obtained from experiments performed using a circular plate loaded on top by granular material (sieved and unsieved sand) and driven to resonance by acoustic wave

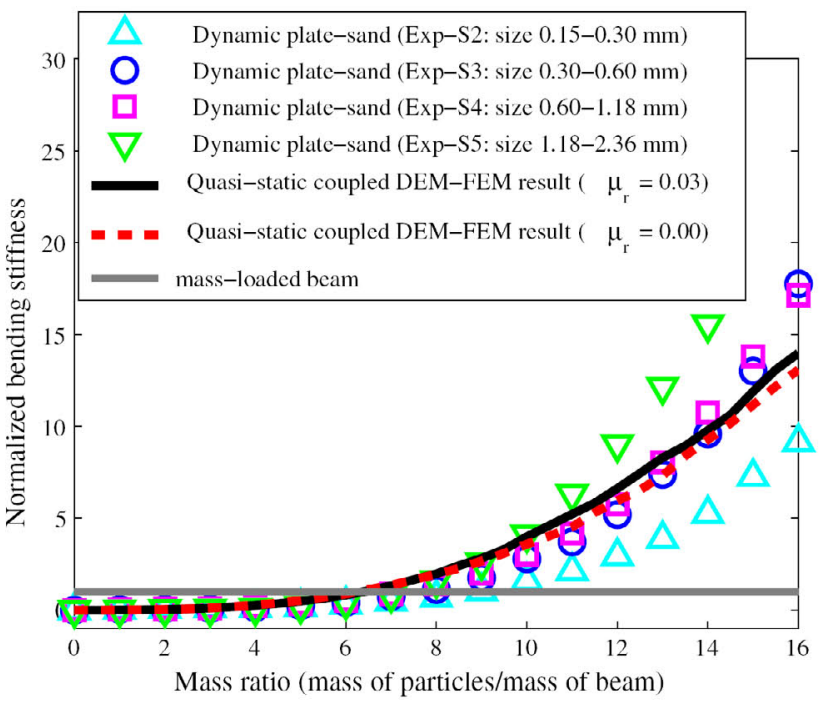

Figure 10. Comparison of resonant frequencies (left) and effective bending stiffness (right) from the 2D quasi-static simulation and the experiments in Kang et al. (2007) for vibration of a plate loaded with sand. The resonant frequencies of the Equivalent-Composite-Beam (ECB) system are normalized by the frequency of the bottom elastic beam (left). The computed effective bending stiffness of the granular layer (or the top beam in the ECB system), normalized by the bending stiffness of the bottom beam, for the case with and without rolling resistance are compared to the experimental results from Kang et al. (2007), which are normalized by the bending stiffness of the supporting plate (right). For comparison, the bending stiffness of the bottom beam is shown (gray horizontal line). 
sweep. As the granular mass loading the beam increases, the thickness of the granular layer does too, and this provides additional bending stiffness to that of the supporting beam. The mechanism responsible for this phenomenon is the behavior of the force chains, shown in the section above. An increased stiffness of the GLEB system results in a higher resonant frequency compared to the case when the supporting beam is loaded by a mass with no stiffness (such as a liquid), which we call a "mass-loaded" beam (see Figure 10). We observe that the resonant frequencies fall into two regimes: "mass-dominant" regime, up to mass ratio $\sim 4$, and "bending-stiffness" regime, for mass ratio $>4$. Even if our present computations are done in 2D and quasi-static bending deformation, the coordination with the 3D experimental results obtained from dynamic resonance is remarkable. In the mass-dominant regime, the frequency decreases as the mass increases with no addition in bending stiffness. In contrast, for the bendingstiffness regime, the frequency increases as the thickness (mass ratio) increases resulting in a stiffer system. As seen from our DEM-FEM computations coupled with the inverse problem determination of the elastic modulus of the top equivalent beam (Figure 10-left) the rolling resistance gives a slightly higher resonant frequency than the case without rolling resistance as the layer becomes thicker.

In contrast with the 3D dynamic experimental data (Kang et al., 2007), our 2D DEM-FEM quasi-static simulations show that the resonant frequencies continue to increase as the thickness increases whereas the experimental values show a leveling of the frequencies for larger mass ratios (thicker layers) especially for the bigger particle sizes. One of the explanations for the observed experimental results is that once the granular layer becomes too thick, driving it into resonance requires forcing amplitudes that lead to nonlinear response which is difficult to process in the same manner as the one described in Kang et al. (2007).

In Figure 10-right we plot the bending stiffness of the top elastic beam in the ECB system or the effective bending stiffness of the granular layer as a function of the layer thickness (or mass ratio). The experimental results from Kang et al. (2007) are also shown for comparison. The calculation of the bending stiffness of the granular layer (top beam) is based on the beam thickness: $D_{1}=E_{1} I_{1}\left(H_{1}\right)$. We note that our computed bending stiffness of the granular layer compares well with the experimental values obtained via the 3D dynamic plate tests. In Figure 10-right, we can see that the granular layer in our model becomes stiffer than the elastic beam alone as the mass ratio becomes larger than 6 . Also, the difference in the granular layer bending stiffness is observed between the two cases: with and without rolling resistance, especially when mass ratios become larger than 10 .

We conclude that the coupled DEM-FEM model is able to capture changes in the structure of the force chains and the shifts in the resonant frequencies of the granularlayer/elastic-beam (GLEB) system with increasing granular layer thickness under the quasi-static bending simulations. The experimental evidence in Kang et al. (2007) points towards a discrepancy between the measured res- onant frequency particle-size dependence of the granular layer and the one predicted by well-bonded static analytical 3D models. For additional insight into why effective media theories may fail for granular materials see Makse et al. (1999). The quasi-static results above lead to a particle-size dependence that matches the analytical model based on 2D contacts. This is discussed elsewhere (Rattanadit, 2009; Rattanadit et al., 2009). For a possible prediction of the experimentally-observed particle-size dependence reported in Kang et al. (2007) and explanation of the discrepancy with the analytical values given by well-bonded effective media models, we need to model a dynamic vibration of the GLEB system and test if the stiffening (changes in the resonant frequency) with increasing thickness of the layer takes place. In the next section we investigate, dynamically, the behavior of the GLEB system under free vibrations.

\subsection{Structure of force chains and resonant behavior of the GLEB system under free vibrations}

The structure changes in force chains and resonant behavior of the granular-layer/elastic-beam (GLEB) system under free bending vibration are investigated in this section. We allow the system to reach the equilibrium from the following initial configuration: the beam is straight and the particles are arranged (in close packed form) on top of it. The system starts vibrating (free vibrations) with small amplitudes and reaches equilibrium before $0.5 \mathrm{~s}$. In Figure 11 we plot the vertical position of the beam's mid-point as a function of time for the two cases: with and without rolling resistance. Notice that the beam never bends above its initial straight position. Similar to the quasi-static (bending deformation) case, we consider the structural changes in the force chains for the thickest granular layer (mass ratio $~ 35.50$ ) and the same material parameters are given in Table 1. Snapshots of the force chains for the cases with (left side) and without (right) rolling resistance are shown in Figure 12.

In contrast to the quasi-static behavior, Figure 12 shows that there are no reversals of the arches forming at equilibrium. As in the quasi-static bending, the intensity of the force chains is higher when rolling resistance is included and the region in the granular layer just above the mid-point of the beam is under very low pressure. From the small free bending vibrations we extract the first natural frequency of the GLEB system, for various granular layer thicknesses, for the two cases: with and without rolling resistance.

We now investigate the behavior of resonant frequency as a function of the granular layer thickness (or mass ratio) for with and without rolling resistance. In 3D dynamic resonant experiments by Kang et al. (2007), the shift in resonant frequencies and stiffness effect is observed (see Figure 10). Here, we simulate a $2 \mathrm{D}$ version of the $3 \mathrm{D}$ experiments performed in Kang et al. (2007). In experiments, a frequency sweep is used through an acoustic speaker to drive a plate loaded by a granular layer (sand) into resonance.

From the free vibration response amplitude (the timedomain displacement of the supporting beam in the GLEB 


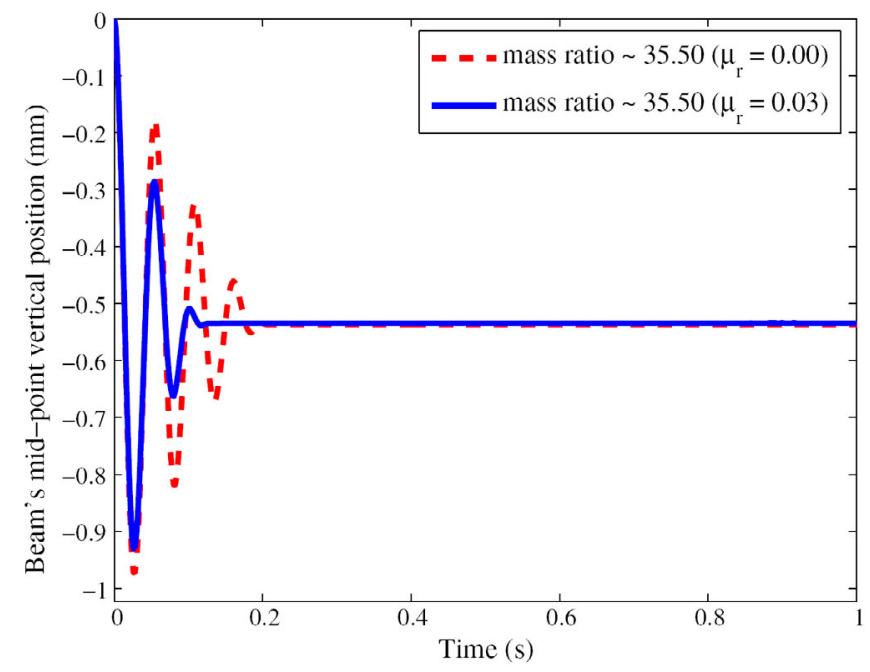

Figure 11. The beam's mid-point vertical position of the granular-layer/elastic-beam system for a mass ratio of 35.50 under free vibrations; notice that rolling resistance dampens the systems and equilibrium is reached faster compared to the case with no rolling resistance.
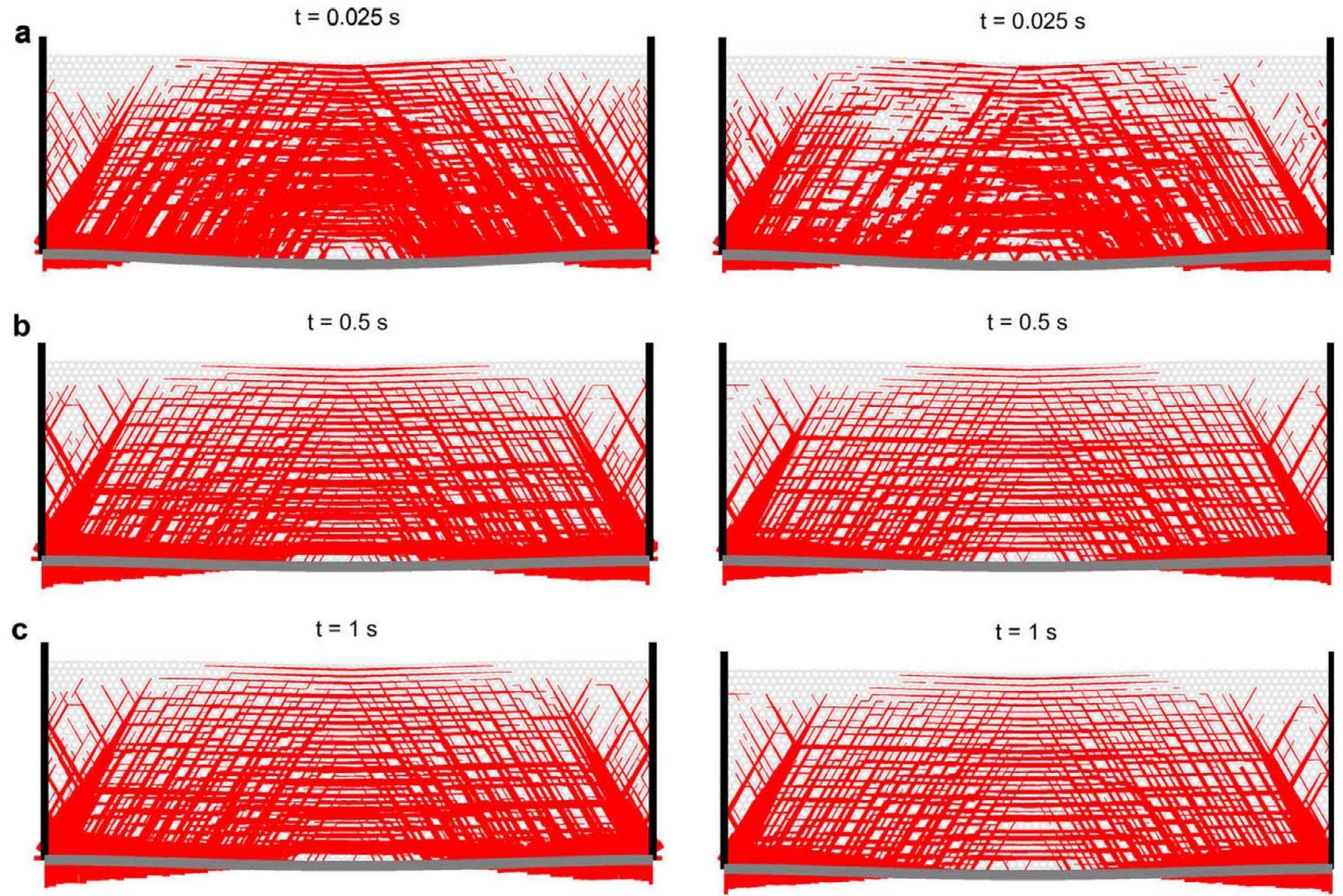

Figure 12. Snapshots of the contact force chains (red color) for the granular-layer/elastic-beam system under free vibrations at $t=0.025$, 0.5 , and $1.0 \mathrm{~s}$; with rolling resistance (left column); without rolling resistance (right column). Note that the particles, walls, and the beam are drawn by light gray, black and gray colors, respectively.

system) computed by the 2D DEM-FEM code, we obtain the first bending resonant frequency by transforming this response in time domain into the response amplitude in the frequency domain, using the Fast-Fourier-Transform
(FFT) as follows: to recover the first resonant bending frequency and eliminate the possibility of higher modes interference, we average the vertical displacement of all beam nodes; we apply the FFT on this averaged displace- 

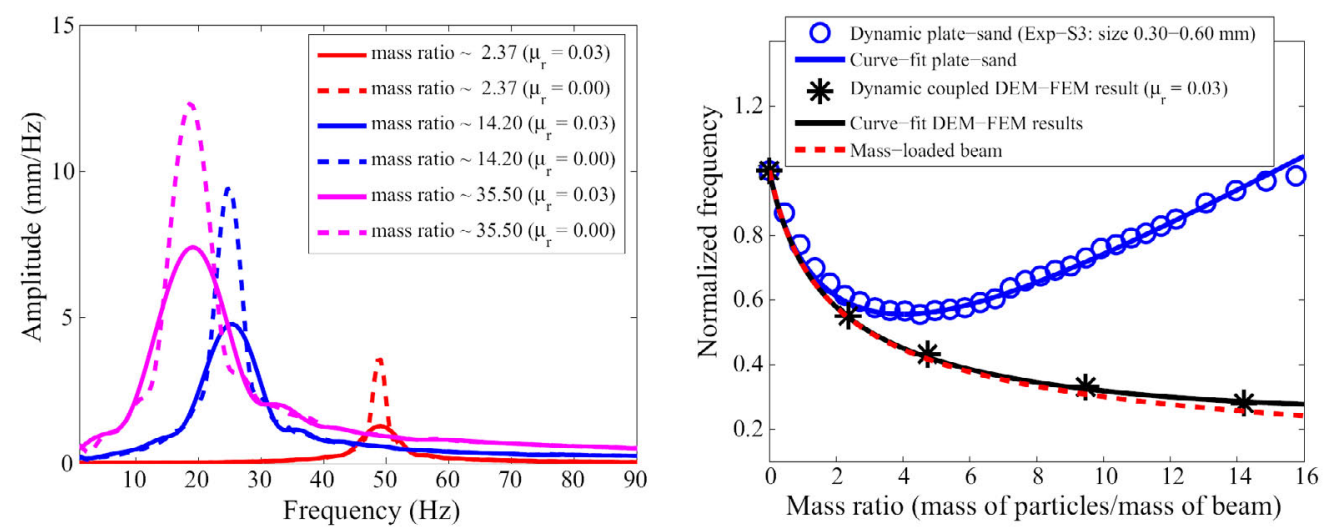

Figure 13. Resonant frequencies (left figure) from the 2D coupled DEM-FEM model for mass ratios of 2.37, 14.20 and 35.50 with rolling resistance (dashed lines) and without rolling resistance (solid lines). Comparison of normalized resonant frequencies (right figure) from the experimental data for sand of size $0.3 \sim 0.6 \mathrm{~mm}$, the 2D coupled DEM-FEM model, and the mass-loaded beam.

ment to obtain the frequency response of the system. We compute this data for five different thicknesses or mass ratios of $2.37,4.73,9.47,14.20$, and 35.50 of the granular layer on top of the beam, for both cases: with and without rolling resistance. The amplitude responses in the frequency domain for the mass ratios of 2.37, 14.20, and 35.50 are shown in Figure 13-left. The peaks in these responses identify the resonant frequencies for each thickness and each case: with or without rolling resistance. The peak amplitude response is lower in the presence of rolling resistance (see Figure 13-left). This is to be expected since rolling resistance increases system's energy dissipation (in rotation). Also, we note that the rolling resistance plays no role in shifting/changing the resonant frequency values under this free-vibration dynamic regime. Figure 13-right shows that the normalized frequencies obtained from our 2D free-vibration simulation align with the results from the mass-loaded analytical results.

The 3D dynamic experimental results are also shown in Figure 13 for qualitative comparison only. Thus, the freevibration under self-weight leads to no stiffening effect, no matter how large is the thickness of the granular layer on top of the beam. We conjecture that the reason for the observed behavior (that contrast the 3D dynamic experimental results in Kang et al. (2007) where the increase in bending stiffness with increasing thickness of the granular layer is observed) is that no changes take place in the structure of the force chains under the small, free-vibration regime. The reason for which the quasi-static simulation (push-up bending) gave results similar to the experiments was the reversal of the self-forming arches when the force chains start pushing against the walls of the contained and against the supporting beam. The problem of forced vibrations of the GLEB system is treated in Rattanadit (2009) and Rattanadit et al. (2009).

\section{Conclusions}

We developed coupled 2D Discrete Element Method-Finite Element Methods (DEM-FEM) simulations to analyze, for the first time, the behavior of granular layers un- der bending deformation. We validated our code for the dynamic case of one particle on top of elastic beam using a FEM-only code (ABAQUS). For a granular-layer/elastic-beam (GLEB) system, we analyzed the structure and changes taking place in the force chains during a slow-dynamics (quasi-static) push-up bending of the system. The behavior of the force chains during bending leads to the possibility of enhanced mixing in vibrated granular media (Promratana, 2008).

We used an analogy with a composite elastic beam to extract, from the computed DEM-FEM response, the Young's modulus of the corresponding granular layer. The values are in the range of 5-8 MPa for the case when rolling resistance is considered and 3-8 $\mathrm{MPa}$ when rolling resistance is absent. These values are in the similar range with those obtained from the experiments of Yanagida et al. (2003) and Kang et al. (2007) and they are significantly lower than those expected from wave speed measurements (Johnson, 1985; Oelze et al., 2002).

From the Young's modulus values, we determined the first resonant bending frequencies of the Equivalent-Composite-Beam (ECB) system, which is analog to our GLEB system, for various thicknesses of the granular layer on top of the elastic beam. We also computed the corresponding bending stiffness of the granular layer alone. The values obtained match, qualitatively, remarkably well with experimental results conducted in 3D experiments on sand on top of an elastic circular plate (Kang et al., 2007) even if the contact model in $2 \mathrm{D}$ is different from that in 3D. There is an increase in the granular layer bending stiffness with an increase in the layer thickness. The evolution of the force chain structures show that the stiffening effect can be explained by the reversal of the selfforming force chain arches, which, instead of resting on the supporting beam, now (when the system is bent upwards) push against the side-walls and the middle of the beam.

For a possible explanation of the discrepancy between the analytical values given by well-bonded effective media models for the particle-size dependence of the resonant frequency of the GLEB system and those obtained 
in experiments (see Kang et al., 2007), we modeled the free vibration dynamics under self-weight. Surprisingly, the systems response follows that of a mass-loaded beam, with no contribution to the bending stiffness coming from the granular layer. We explain this by observing that, under the free vibrations, the amplitude of the motion is small and it never leads to bending upward as in the quasi-static case. The reversal of the force chain arches does not take place. Analysis of forced-vibrations of the GLEB system is planned in future work.

\section{Acknowledgments}

The research was sponsored by the U.S. Army Research Laboratory under the RMAC-RTP Cooperative Agreement No. W911NF-04-2-0011.

\section{References}

Antony, 2007 - S. J. Antony, Link between single-particle properties and macroscopic properties in particulate assemblies: role of structures within structures, Philosophical Transactions of the Royal Society A 365 (2007), pp. 2879-2891.

Asmar et al., 2002 • B. N. Asmar, P. A. Langston, AJ. Matchett and J. K. Walters, Validation tests on a distinct element model of vibrating cohesive particle systems, Computers and Chemical Engineering 26 (2002), pp. 785-802.

Baxter et al., $1997 \bullet$ J. Baxter, U. Tuzun, J. Burnell and D. M. Heyes, Granular dynamics simulations of two-dimensional heap formation, Physical Review E 55 (1997), pp. 3546-3554.

Brilliantov and Poschel, $1998 \bullet$ N. V. Brilliantov and T. Poschel, Rolling friction of a viscous sphere on a hard plane, Europhysics Letters 42 (1998), pp. 511-516.

Campbell, 1997 • C. S. Campbell, Computer simulation of powder flows. In: Gotoh et al., Editors, Powder Technology Handbook (2nd ed. ), Dekker, New York (1997), pp. 777-793.

Campbell, 2006 • C. S. Campbell, Granular material flows -an overview, Powder Technology 162 (2006), pp. 208-229.

Cleary, $1998 \bullet$ P. W. Cleary, Predicting charge motion, power draw, segregation and wear in ball mills using discrete element methods, Minerals Engineering 11 (1998), pp. 1061-1080.

Cleary and Sawley, 2002 • P. W. Cleary and M. L. Sawley, DEM modelling of industrial granular flows: 3D case studies and the effect of particle shape on hopper discharge, Applied Mathematical Modelling 26 (2002), pp. 89-111.

Cundall and Strack, $1979 \bullet$ P. A. Cundall and O. D. L. Strack, A discrete numerical model for granular assemblies, Geotechnique 29 (1979), pp. 47-65.

Frangin et al., 2006 • E. Frangin, P. Marin and L. Daudeville, On the use of combined finite/discrete element method for impacted concrete structures, Journal de Physique IV 134 (2006), pp. 461-466.

Gerl and Zippelius, 1999 • F. Gerl and A. Zippelius, Coefficient of restitution for elastic disks, Physical Review E 59 (1999), pp. 2361-2372.

Gethin et al., 2006 • D. T. Gethin, X. S. Yang and R. W. Lewis, A two dimensional combined discrete and finite element scheme for simulating the flow and compaction of systems comprising irregular particulates, Computer Methods in Applied Mechanics and Engineering 195 (2006), pp. 5552-5565.
Haff and Werner, 1986 • P. K. Haff and B. T. Werner, Computer simulation of the mechanical sorting of grains, Powder Technology 48 (1986), pp. 239-245.

Han et al., 2000a • K. Han, D. Peric, A. J. L. Crook and D. R. J. Owen, A combined finite-discrete element simulation of shot peening processes Part I: studies on 2D interaction laws, Engineering Computations 17 (5) (2000), pp. 593-619.

Han et al., 2000b • K. Han, D. Peric, D. R. J. Owen and J. Yu, A combined finite/discrete element simulation of shot peening processes Part II: 3D interaction laws, Engineering Computations 17 (6) (2000), pp. 680-702.

Hertz, $1882 \bullet$ H. Hertz, 1882. Uber die Beruhrung fester elastische Korper and uber die Harts (On the contact of rigid elastic solids and on hardness), Verhandlunger des Vereins zur Beforderung des Gewerbefleisses, Leipzig.

Ji and Shen, $2006 \bullet$ S. Ji and H. H. Shen, Effect of contact force models on granular flow dynamics, Journal of Engineering Mechanics 132 (2006), pp. 1252-1259.

Johnson, $1985 \bullet$ K. L. Johnson, Contact Mechanics, Cambridge University Press, Berlin (1985).

Kang, $2006 \bullet$ W. Kang, 2006. Vibration of Plates Loaded with Granular Materials. M.S. Thesis. University of NebraskaLincoln, Department of Engineering Mechanics.

Kang et al., $2007 \bullet$ W. Kang, J. A. Turner, F. Bobaru, L. Yang and K. Rattanadit, Granular layers on vibrating plates: Effective bending stiffness and particle-size effects, Journal of the Acoustical Society of America 121 (2007), pp. 888-896.

Komodromos and Williams, $2004 \bullet$ P. Komodromos and R. Williams, Dynamic simulation of multiple deformable bodies using combined discrete and finite element methods, Engineering Computations 21 (2004), pp. 431-448.

Komodromos, $2005 \bullet$ P. Komodromos, A simplified updated Lagrangian approach for combining discrete and finite element methods, Computational Mechanics 35 (2005), pp. 305-313.

Kruggel et al., $2007 \bullet$ H. Kruggel, E. Simsek, S. Rickelt, S. Wirtz and V. Scherer, Review and extension of normal force models for the discrete element method, Powder Technology 171 (2007), pp. 157-173.

Kudrolli, $2004 \bullet$ A. Kudrolli, Size separation in vibrated granular matter, Reports on Progress in Physics 67 (2004), pp. 209-247.

Kuninaka and Hayakawa, $2001 \bullet$ H. Kuninaka and H. Hayakawa, The impact of two-dimensional elastic disk, Journal of the Physical Society of Japan 70 (2001), pp. 2220-2221.

Kuo et al., $2002 \bullet$ H. P. Kuo, P. C. Knight, D. J. Parker, Y. Tsuji, M. J. Adams and J. P. K. Seville, The influence of DEM simulation parameters on the particle behaviour in a V-mixer, Chemical Engineering Science 57 (2002), pp. 3621-3638.

Langston and Tuzun, $1994 \bullet$ P. A. Langston and U. Tuzun, Continuous potential discrete particle simulations of stress and velocity fields in hoppers: transition from fluid to granular flow, Chemical Engineering Science 49 (1994), pp. 1259-1275.

Langston et al., 1995 • P. A. Langston, U. Tuzun and D. M. Heyes, Discrete element simulation of granular flow in 2D and 3D hoppers: Dependence of discharge rate and wall stress on particle interactions, Chemical Engineering Science 50 (1995), pp. 967-987.

Lewis et al., $2005 \bullet$ R. W. Lewis, D. T. Gethin, X. S. Yang and R. C. Rowe, A combined finite-discrete element method for simulating pharmaceutical powder tableting, International Journal for Numerical Methods in Engineering 62 (2005), pp. 853-869. 
Makse et al., $1999 \bullet$ H. A. Makse, N. Gland, D. L. Johnson and L. M. Schwartz, Why effective medium theory fails in granular materials, Physical Review Letters 83 (24) (1999), pp. 5070-5073.

Mindlin and Deresiewicz, 1953 • R. D. Mindlin and H. Deresiewicz, Elastic spheres in contact under varying oblique forces, ASME Journal of Applied Mechanics 20 (1953), pp. $327-344$.

Oelze et al., 2002 • M. L. Oelze, W. D. O'Brien and R. G. Darmody, Measurement of attenuation and speed of sound in soils, Soil Science Society of America Journal 66 (2002), pp. 788-796.

Onate and Rojek, 2004 • E. Onate and J. Rojek, Combination of discrete element and finite element methods for dynamic analysis of geomechanics problems, Computer Methods in Applied Mechanics and Engineering 193 (2004), pp. 3087-3128.

Petrinic, $1996 \bullet$ N. Petrinic, 1996. Aspects of discrete element modeling involving facet-to-facet contact detection and interaction. Ph.D. Thesis. University of Wales, Swansea.

Poritsky, $1950 \bullet \mathrm{H}$. Poritsky, Stresses and deflections of cylindrical bodies in contact with application to contact of gears and locomotive wheels, Journal of Applied Mechanics 17 (1950), pp. 191-201.

Poschel and Schwager, $2005 \bullet$ T. Poschel and T. Schwager, Computational Granular Dynamic: Models and Algorithms, Springer (2005).

Promratana, 2008 • K. Promratana, 2008. Granular Materials Behavior under Dynamic Excitations. M.S. Thesis. University of Nebraska-Lincoln, Department of Engineering Mechanics, December 2008.

Rattanadit, 2009 • K. Rattanadit, 2009. Coupled DEM-FEM Simulations for Analysis of Quasi-static and Dynamic Properties of Granular Systems on Elastic Foundation. Ph.D. Thesis. University of Nebraska-Lincoln, Department of Engineering Mechanics, May 2009.

Rattanadit et al., $2009 \bullet$ K. Rattanadit, F. Bobaru, K. Promratana, J. A. Turner, 2009. Particle size-dependence of the bending stiffness in granular layers on elastic foundations using a coupled DEM-FEM approach, to be submitted for publication.

Reddy, 2003 - J. N. Reddy, Introduction to the Finite Element Method, McGraw-Hill (2003).

Renzo and Maio, 2004 - A. D. Renzo and F. P. Maio, Comparison of contact force models for the simulation of collisions in DEM based granular flow codes, Chemical Engineering Science 59 (2004), pp. 525-541.

Sebastian and Luis, $2005 \bullet$ L. G. Sebastian and V. Luis, Discrete element method evaluation of granular crushing under direct shear test conditions, Journal of Geotechnical and Geoenvironmental Engineering 131 (2005), pp. 1295-1300.

Schafer et al., $1996 \bullet$ J. Schafer, S. Dippel and D. E. Wolf, Force schemes in simulations of granular materials, J. Phys. I France 6 (1996), pp. 5-20.
Taguchi, $1992 \bullet \mathrm{Y}$. Taguchi, New origin of convective motion: elastically induced convection in granular materials, Physical Review Letters 69 (1992), pp. 1367-1370.

Thompson and Grest, $1991 \bullet$ P. A. Thompson and G. S. Grest, Granular flow: friction and the dilatancy transition, Physical Review E 67 (1991), pp. 1751-1754.

Thornton and Randall, $1988 \bullet$ C. Thornton and C. W. Randall, Applications of theoretical contact mechanics to solid particle system simulation. In: M. Satake and J. T. Jenkins, Editors, Micromechanics of Granular Materials, Elsevier, Amsterdam (1988), pp. 133-142.

Thornton and Yin, 1991 • C. Thornton and K. K. Yin, Impact of elastic spheres with and without adhesion, Powder Technology 65 (1991), pp. 153-166.

Tijskens et al., 2003 • E. Tijskens, H. Ramon and J. Baerdemaeker, Discrete element modeling for process simulation in agriculture, Journal of Sound and Vibration 266 (2003), pp. 493-514.

Tsuji et al., $1993 \bullet$ Y. Tsuji, T. Kawaguchi and T. Tanaka, Discrete particle simulation of two-dimensional fluidized bed, Powder Technology 77 (1993), pp. 79-87.

$\mathrm{Xu}$ and $\mathrm{Yu}, 1997 \bullet$ B. H. Xu and A. B. Yu, Numerical simulation of the gas-solid flow in a fluidized bed by combining discrete particle method with computational fluid dynamics, Chemical Engineering Science 52 (1997), pp. 2785-2809.

$\mathrm{Xu}$ and $\mathrm{Wu}, 2007 \bullet \mathrm{R}$. $\mathrm{Xu}$ and $\mathrm{Y}$. Wu, Static, dynamic, and buckling analysis of partial interaction composite members using Timoshenko's beam theory, International Journal of Mechanical Sciences 49 (2007), pp. 1139-1155.

Yamane, 2004 - K. Yamane, Discrete-element method application to mixing and segregation model in industrial blending system, Journal of Materials Research 19 (2004), pp. 623-627.

Yanagida et al., $2003 \bullet$ T. Yanagida, A. J. Matchett, B. N. Asmar, P. A. Langston, J. K. Walters and J. M. Coulthard, Dynamic response of well-mixed binary particulate systems subjected to low magnitude vibration, Advanced Powder Technology 14 (2003), pp. 589-604.

Zhang and Whiten, 1996 • D. Zhang and W. J. Whiten, The calculation of contact forces between particles using spring and damping models, Powder Technology 88 (1996), pp. 59-64.

Zhou et al., 2003 • Y. C. Zhou, B. H. Xu, R. P. Zou, A. B. Yu and P. Zulli, Stress distribution in a sandpile formed on a deflected base, Advanced Powder Technology 14 (4) (2003), pp. 401-410.

Zhou et al., $1999 \bullet$ Y. C. Zhou, W. D. Wright, R. Y. Yang, B. H. $\mathrm{Xu}$ and $\mathrm{A}$. B. Yu, Rolling friction in the dynamic simulation of sandpile formation, Physica A 269 (1999), pp. 536-553.

Zhu and Yu, $2006 \bullet$ H. P. Zhu and A. B. Yu, A theoretical analysis of the force models in discrete element method, Powder Technology 161 (2006), pp. 122-129. 